\title{
Planck pre-launch status: The HFI instrument, from specification to actual performance
}

J.-M. Lamarre' ${ }^{1}$, J.-L. Puget ${ }^{2}$, P. A. R. Ade ${ }^{3}$, F. Bouchet ${ }^{4}$, G. Guyot ${ }^{2}$, A. E. Lange ${ }^{5,6, \dagger}$, F. Pajot ${ }^{2}$, A. Arondel ${ }^{2}$, K. Benabed ${ }^{4}$, J.-L. Beney ${ }^{8}$, A. Benoît ${ }^{9}$, J.-Ph. Bernard ${ }^{10}$, R. Bhatia ${ }^{7}$, Y. Blanc ${ }^{11}$, J. J. Bock ${ }^{5,6}$, E. Bréelle ${ }^{12}$, T. W. Bradshaw ${ }^{13}$, P. Camus ${ }^{9}$, A. Catalano ${ }^{12,1}$, J. Charra ${ }^{2, \dagger}$, M. Charra ${ }^{2}$, S. E. Church ${ }^{14}$, F. Couchot ${ }^{8}$, A. Coulais ${ }^{1}$, B. P. Crill ${ }^{5,6}$, M. R. Crook ${ }^{13}$, K. Dassas ${ }^{2}$, P. de Bernardis ${ }^{15}$, J. Delabrouille ${ }^{12}$, P. de Marcillac ${ }^{2}$, J.-M. Delouis ${ }^{4}$, F.-X. Désert ${ }^{16}$, C. Dumesnil ${ }^{2}$, X. Dupac ${ }^{17}$, G. Efstathiou ${ }^{18}$, P. Eng ${ }^{2}$, C. Evesque ${ }^{2}$, J.-J. Fourmond ${ }^{2}$, K. Ganga ${ }^{12}$, M. Giard ${ }^{10}$, R. Gispert ${ }^{2, \dagger}$, L. Guglielmi ${ }^{12}$, J. Haissinski ${ }^{8}$, S. Henrot-Versillé ${ }^{8}$, E. Hivon ${ }^{4}$, W. A. Holmes ${ }^{6}$, W. C. Jones ${ }^{6,19}$, T. C. Koch ${ }^{6}$, H. Lagardère ${ }^{2}$, P. Lami ${ }^{2}$, J. Landé ${ }^{10}$, B. Leriche ${ }^{2}$, C. Leroy ${ }^{2}$, Y. Longval ${ }^{2}$, J. F. Macías-Pérez ${ }^{20}$, T. Maciaszek ${ }^{11}$, B. Maffei ${ }^{21}$, B. Mansoux ${ }^{8}$, C. Marty ${ }^{10}$, S. Masi ${ }^{15}$, C. Mercier ${ }^{2}$, M.-A. Miville-Deschênes ${ }^{2}$, A. Moneti ${ }^{4}$, L. Montier ${ }^{10}$, J. A. Murphy ${ }^{22}$, J. Narbonne ${ }^{10}$, M. Nexon ${ }^{10}$, C. G. Paine ${ }^{6}$, J. Pahn ${ }^{11}$, O. Perdereau ${ }^{8}$, F. Piacentini ${ }^{15}$, M. Piat ${ }^{12}$, S. Plaszczynski ${ }^{8}$, E. Pointecouteau ${ }^{10}$, R. Pons ${ }^{10}$, N. Ponthieu ${ }^{2}$, S. Prunet ${ }^{4}$, D. Rambaud ${ }^{10}$, G. Recouvreur ${ }^{1}$, C. Renault ${ }^{20}$, I. Ristorcelli ${ }^{10}$, C. Rosset $^{12,8}$, D. Santos ${ }^{20}$, G. Savini ${ }^{3,23}$, G. Serra ${ }^{10, \dagger}$, P. Stassi ${ }^{20}$, R. V. Sudiwala ${ }^{3}$, J.-F. Sygnet ${ }^{4}$, J. A. Tauber ${ }^{7}$, J.-P. Torre ${ }^{2}$, M. Tristram ${ }^{8}$, L. Vibert ${ }^{2}$, A. Woodcraft ${ }^{24}$, V. Yurchenko ${ }^{22,25}$, and D. Yvon ${ }^{26}$

(Affiliations can be found after the references)

Received 24 July 2009 / Accepted 27 January 2010

\section{ABSTRACT}

Context. The High Frequency Instrument (HFI) is one of the two focal instruments of the Planck mission. It will observe the whole sky in six bands in the $100 \mathrm{GHz}-1 \mathrm{THz}$ range.

Aims. The HFI instrument is designed to measure the cosmic microwave background (CMB) with a sensitivity limited only by fundamental sources: the photon noise of the CMB itself and the residuals left after the removal of foregrounds. The two high frequency bands will provide full maps of the submillimetre sky, featuring mainly extended and point source foregrounds. Systematic effects must be kept at negligible levels or accurately monitored so that the signal can be corrected. This paper describes the HFI design and its characteristics deduced from ground tests and calibration.

Methods. The HFI instrumental concept and architecture are feasible only by pushing new techniques to their extreme capabilities, mainly: (i) bolometers working at $100 \mathrm{mK}$ and absorbing the radiation in grids; (ii) a dilution cooler providing $100 \mathrm{mK}$ in microgravity conditions; (iii) a new type of AC biased readout electronics and (iv) optical channels using devices inspired from radio and infrared techniques.

Results. The Planck-HFI instrument performance exceeds requirements for sensitivity and control of systematic effects. During ground-based calibration and tests, it was measured at instrument and system levels to be close to or better than the goal specification.

Key words. cosmic microwave background - space vehicles: instruments - submillimeter: general - techniques: photometric techniques: polarimetric

\section{Introduction}

The main scientific goal of the Planck mission ${ }^{1}$ (Tauber et al. 2010a) is a full sky measurement of the intensity and polarisation anisotropies of the cosmic microwave background (CMB). The High Frequency Instrument (HFI) and the Low Frequency Instrument (LFI) share the focal plane of an off-axis Gregorianlike telescope with an effective diameter of $1.5 \mathrm{~m}$.

Soon after the publication of the COBE results (Mather et al. 1990; Smoot et al. 1991), it was pointed out during an

\footnotetext{
1 Planck (http://www.esa.int/Planck) is a project of the European Space Agency - ESA - with instruments provided by two scientific Consortia funded by ESA member states (in particular the lead countries: France and Italy) with contributions from NASA (USA), and telescope reflectors provided in a collaboration between ESA and a scientific Consortium led and funded by Denmark.
}

internal meeting at IAS (February 1993) that the sensitivity of future receivers in the millimetre range could improve by nearly three orders of magnitude if a number of promising techniques of bolometric detection reached maturity. These were

- (i) the development of $100 \mathrm{mK}$ spider-web bolometers (SWB) (Mauskopf et al. 1997) and, later, of polarisationsensitive bolometers (PSB) (Jones et al. 2003);

- (ii) readout electronics using AC bias currents to suppress low frequency noise and use of total power photometry instead of differential methods (Rieke et al. 1989; Gaertner et al. 1997);

- (iii) a $100 \mathrm{mK}$ dilution cooler operable in microgravity conditions (Benoît et al. 1997);

- (iv) closed cycle refrigerators that could provide temperature stages at both $20 \mathrm{~K}$ and $4 \mathrm{~K}$ without bulky cryostats; 
- (v) an optical design that mixes bolometer and radio techniques.

These solutions had to be incorporated into a new type of architecture to be launched at ambient temperature and able to cool down in space to temperatures appropriate for the bolometers' performance.

The architecture of the HFI had to solve new optical, cryogenic, mechanical and electrical problems that often seemed incompatible. This made the operation of each sub-system dependent on the performance of several others. For example, the one kilogram bolometer plate at $100 \mathrm{mK}$ required rigid positioning to maintain optical alignment, high strength support struts to survive launch accelerations and 60 shielded, twisted pair wiring (one for each bolometer and thermometer and mandatory to suppress electromagnetic interference (EMI)) while conducting only a few $\mu \mathrm{W}$ to be lifted by the dilution cooler. Each of the techniques listed above had to be adapted, within very strict constraints, into a single instrument concept.

The HFI was proposed to the Centre National d'Études Spatiales (CNES) as the focal plane instrument of a dedicated mission called SAMBA, and then as part of a medium mission of the program Horizon 2000 of the European Space Agency (ESA). It was selected for a phase A together with the COBRAS (Mandolesi et al. 1994) proposal. The COBRAS-SAMBA study resulted in the Planck mission that merged into a single telescope the bolometers cooled at $100 \mathrm{mK}$ of SAMBA and the COBRAS $20 \mathrm{~K}$ amplifiers based on the use of high electron mobility transistors. The proposed performance and the expected scientific output of the Planck mission are described in the "Planck Blue Book" (The Planck consortium 2005).

A number of technical developments necessary for HFI were tested in pathfinder ground-based and balloon-borne instruments, like SuZIE (Holzapfel et al. 1997), Diabolo (Benoît et al. 2000), BOOMERANG (Piacentini et al. 2002; Crill et al. 2003; Masi et al. 2006), and MAXIMA (Lee et al. 1999). Essential aspects of the HFI design concept were verified with the balloonborne Archeops instrument (Benoît et al. 2002), whose focal plane instrument closely followed the design of HFI.

This is one of the "Planck pre-launch status" papers. Its purpose is to give an overview of the design that meets the mission requirements and to present the expected HFI performance. More details on HFI specific issues will be found in the companion papers Ade et al. (2010), Maffei et al. (2010), Rosset et al. (2010), Pajot et al. (2010). The Planck mission is presented in Tauber et al. (2010a) and the optics as a system in Tauber et al. (2010b). The next section of this paper is dedicated to the high level scientific requirements that led to the HFI instrument concept, the optical optimisation and the measurement strategy. Section 3 focuses on the sensitivity of the HFI and describes the detection chain from the receivers to the telemetry. Expected sensitivities for the HFI are given in this section. The readout electronics is an essential piece of the detection chain. It is described in Sect. 4 together with the on-board data handling. Section 5 is dedicated to the cryogenic design and to the performance of the HFI coolers, i.e. the open circuit dilution cooler and the $4 \mathrm{~K}$ mechanical cooler and their associated temperature control systems. Section 6 addresses several miscellaneous issues, including the development philosophy and the calibration approach. This last aspect is developped further in Pajot et al. (2010).

\section{HFI architecture and optical optimisation}

\subsection{Design and mission rationale}

The HFI was designed to have a sensitivity limited by the photon noise of the observed radiation, which is possible in the millimetre range with a passively cooled telescope (Lamarre 1986; Lamarre et al. 1995). The main features of the instrument, and of the Planck telescope and the spacecraft, result from this requirement. These initial aspirations for the HFI proved to be achievable following various instrumental developments and the implementation of strict design principles.

The HFI's measurement noise on the CMB is less than the level of contamination expected from the foregrounds, even in clean regions of the sky. A mission concept was proposed to map the whole sky in six bands centred at 100,143, 217, 353, 545 and $857 \mathrm{GHz}$ (Bouchet et al. 1996). The four low frequency bands are dedicated to direct measurement of the CMB and are polarisation sensitive. The two high frequency bands are optimised to identify the foregrounds and to separate them from the CMB. Even with wide spectral coverage, the removal of foregrounds is expected to be the limiting factor for the sensitivity (up to about four times the photon noise limit), and this was adopted as the sensitivity requirement for the HFI. A goal sensitivity of twice the photon noise limit was also set to drive the design of all subsystems and to provide margins in aspects of the instrumentation that had never been explored before at this level of performance. The essential design goals are given in Table 1. Noise equivalent delta temperature and noise equivalent power are goal values, i.e. twice better than the requirement and are consistent with the overall mission goals given in the "Planck Blue Book" (The Planck consortium 2005).

The low frequency limit of the HFI spectral coverage was set mainly by the bolometer technology for very long wavelengths. The merging of the SAMBA concept and the COBRAS proposal (Mandolesi et al. 2010; Bersanelli et al. 2010) extended the spectral range for component separation.

Beginning with its discovery, measurements of the CMB have required control of systematic effects to high precision. The improved sensitivity expected from Planck-HFI requires commensurate improvements in the control of systematic errors. The reduction of systematic effects was a driver of every aspect of the design of the HFI instrument, as described in the following sections.

\subsection{Optical, mechanical and thermal architecture}

The thermal and optical architectures of the focal plane assembly are tightly coupled, because every optical element warmer than $1 \mathrm{~K}$ is a significant source of thermal radiation in the spectral range suitable for CMB measurements. Stray light is also a source of parasitic radiation. Conversely, every optical element absorbs radiation power both from the nominal beam and from stray light. The absorbed heat has to be lifted by the cryogenic stages, and may contribute to their cooling budget. This requires nearly perfect control of stray light and a cascade of optical filters at different temperatures.

The HFI architecture is based on independent optical chains collecting the light from the telescope and feeding it to bolometers or bolometer pairs (for the polarisation-sensitive bolometers, PSBs). Each of the 36 optical chains transmits the desired frequencies and blocks other frequencies with rejection factors up to $10^{10}$. It also reduces the thermal radiation loads on the coldest stage by factors of at least $10^{4}$. Interference mesh filter 
Table 1. HFI design goals. P stands for polarisation sensitive bolometers.

\begin{tabular}{lcccccccccc}
\hline \hline Channel & & $100 \mathrm{P}$ & $143 \mathrm{P}$ & 143 & $217 \mathrm{P}$ & 217 & $353 \mathrm{P}$ & 353 & 545 & 857 \\
\hline Central frequency & $(\mathrm{GHz})$ & 100 & 143 & 143 & 217 & 217 & 353 & 353 & 545 & 857 \\
Bandwidth & $(\%)$ & $33 \%$ & $33 \%$ & $33 \%$ & $33 \%$ & $33 \%$ & $33 \%$ & $33 \%$ & $33 \%$ & $33 \%$ \\
Full width half maximum beam size & $\left({ }^{\prime}\right)$ & 9.6 & 7.0 & 7.0 & 5.0 & 5.0 & 5.0 & 5.0 & 5.0 & 5.0 \\
Number of bolometers & & 8 & 8 & 4 & 8 & 4 & 8 & 4 & 4 & 4 \\
NE $\Delta T_{\mathrm{CMB}}$ per bolometer & $\left(\mu \mathrm{K}_{\mathrm{CMB}} \mathrm{s}^{1 / 2}\right)$ & 100 & 82 & 62 & 132 & 91 & 404 & 277 & 2000 & 91000 \\
$\mathrm{NE} \Delta T_{R-J}$ per bolometer & $\left(\mu \mathrm{K}_{R-J} \mathrm{~S}^{1 / 2}\right)$ & 77 & 50 & 38 & 45 & 31 & 34 & 23 & 14 & 9.4 \\
Bolometer NEP & $\left(a \mathrm{Ws} \mathrm{s}^{1 / 2}\right)$ & 10.6 & 9.7 & 14.6 & 13.4 & 18.4 & 16.4 & 22.5 & 72.3 & 186 \\
\hline
\end{tabular}
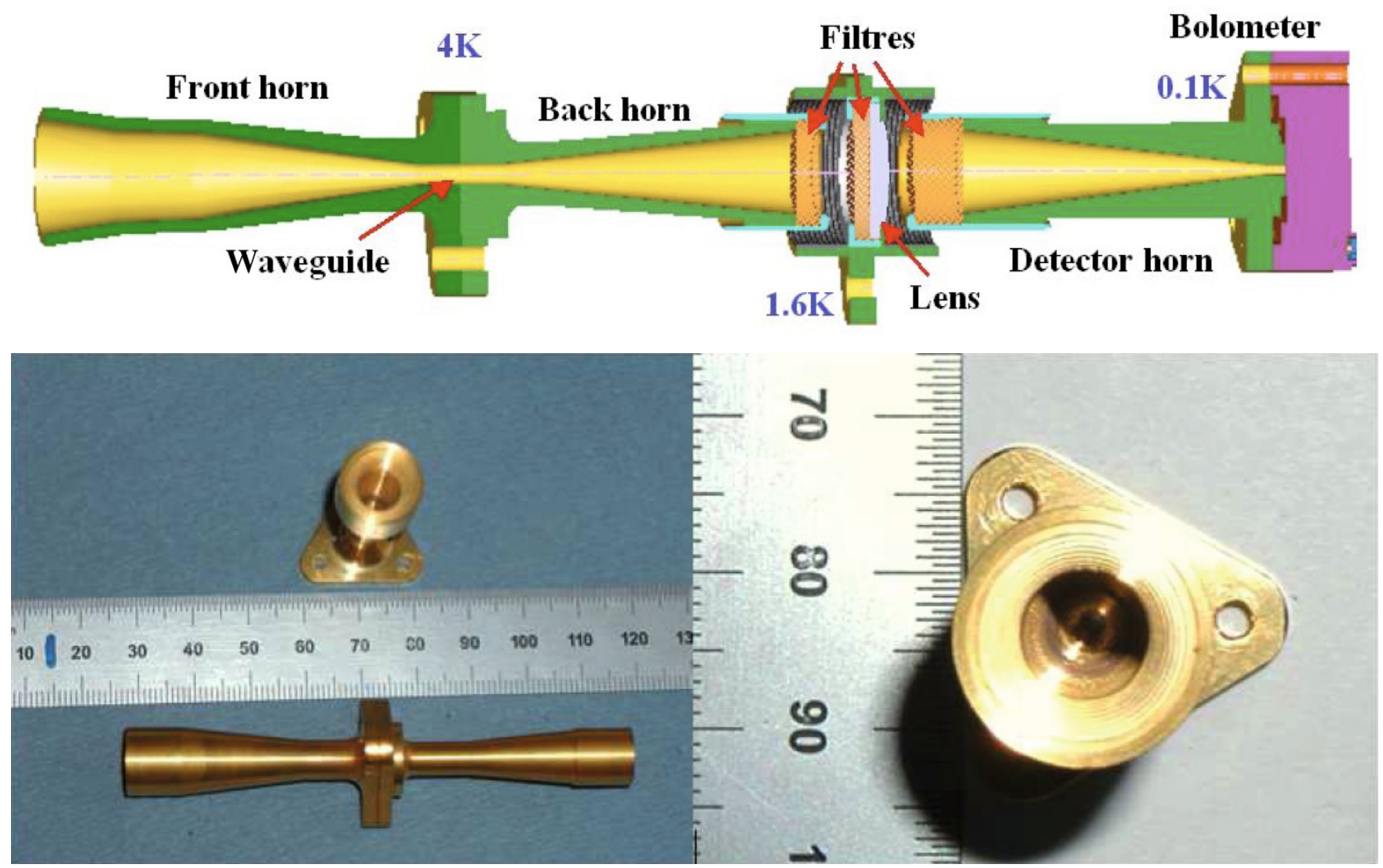

Fig. 1. A typical optical chain. The reference scale is in $\mathrm{mm}$.

and waveguide sections provide the spectral and thermal performance (Ade et al. 2010), while corrugated horns aligned with each other propagate the radiation with the required geometry (Maffei et al. 2010). Sixteen out of the 36 channels feed pairs of PSBs. The external horn defines the illumination of the telescope and therefore the angular resolution of each channel on the sky.

A typical optical chain is shown in Fig. 1. At $4 \mathrm{~K}$, the backto-back horns provide initial geometrical and spectral selection of the radiation, and a first set of filters blocks the highest frequency and most energetic part of the background. Interference filters that show a high efficiency in the transmission band are particularly well adapted to our needs, since their thermal emission is minimum inside and outside the band. They are used on the $1.6 \mathrm{~K}$ stage and at the entrance of the $0.1 \mathrm{~K}$ stage, where they define the high frequency limit of the band and block the thermal emission of the warmer stages.

In order to ensure proper positioning and cooling, these elements are attached onto three enclosures in a nested arrangement, as shown in Fig. 2. They are called the $4 \mathrm{~K}, 1.6 \mathrm{~K}$ and $0.1 \mathrm{~K}$ stages, and their real operating temperatures are about $4.5 \mathrm{~K}, 1.4 \mathrm{~K}$, and $0.1 \mathrm{~K}$ respectively. Each enclosure is lighttight, aside from the well-defined and filtered flux in the optical chains. All stages are thermally isolated from each other, while rigidly and accurately positioned with respect to each other and to the telescope with rigid and thermally isolating materials. Active control of the temperature of the three stages keeps their temperature stable enough so that variations of their thermal emission are negligible (Piat et al. 2003). The cryogenic and thermal designs are detailed in Sect. 5.

\subsection{Spectral selection}

The six spectral bands of the HFI instrument (Table 1) cover in a contiguous manner all frequencies from $84 \mathrm{GHz}$ up to $1 \mathrm{THz}$ via adjacent bands with close to $33 \%$ relative bandwidth. This provides multiple frequency coverage whilst maximizing 


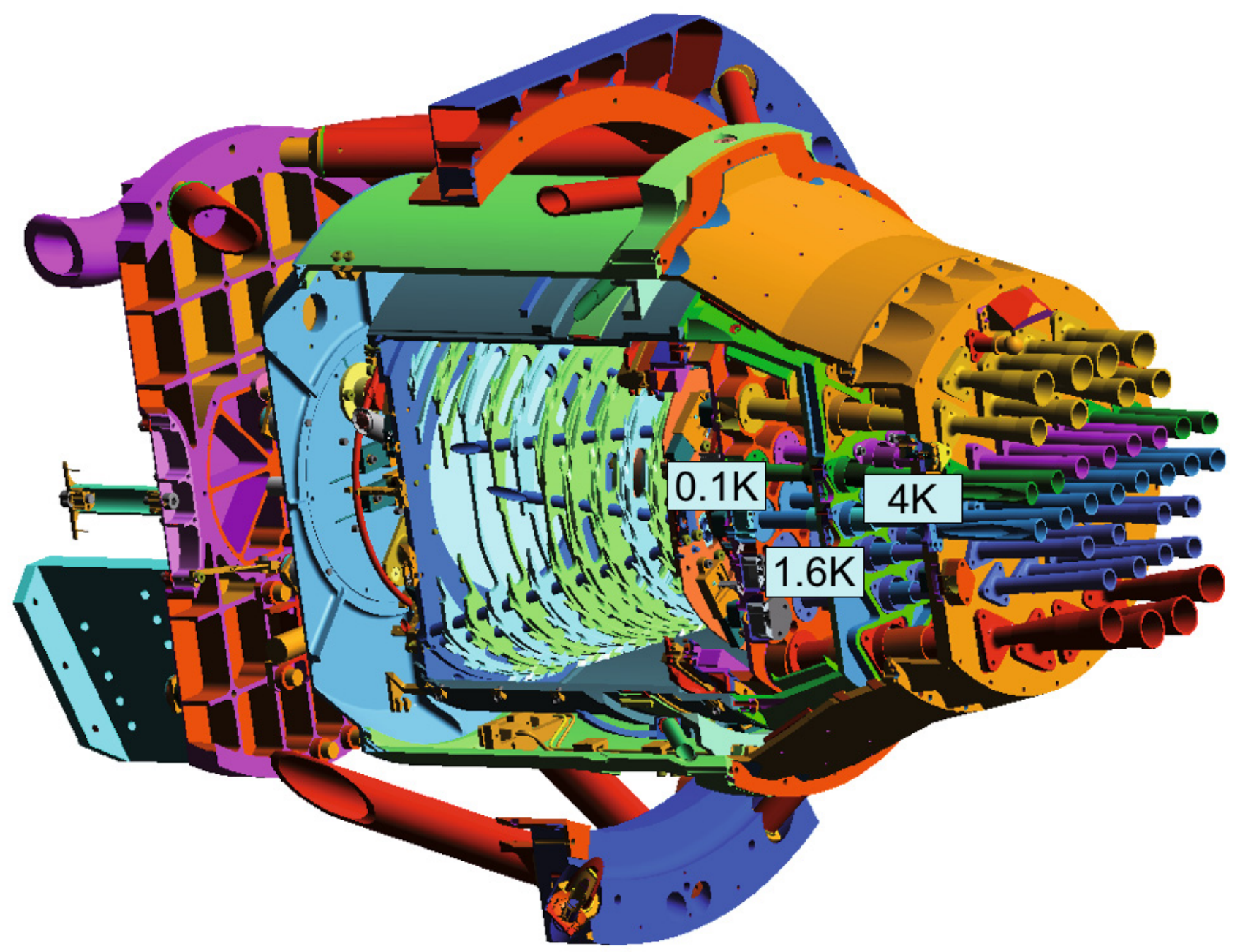

Fig. 2. The Russian doll arrangement of the HFI focal plane unit.

photon collection efficiency. Out of band rejection in each band is achieved with waveguide extinction on the low frequency side for all the single moded channels and with a high-pass filter in the multi-moded channels. A set of low-pass filters provides the high frequency rejection. This is not only to ensure that rejection of higher energy photons is well within specification, but also to guarantee (especially in the higher frequency pixels) that contamination from the $\mathrm{CMB}$ is minimal in channels designed to be mostly sensitive to foreground signals (Ade et al. 2010).

\subsection{The stray light versus angular resolution trade-off}

The beam patterns of the horns select the radiation that reaches the bolometers. Their shape determines the effective aperture of the telescope for this horn and then the angular resolution on the sky set by beam propagation laws. A large effective aperture gives an optimised aperture efficiency (Kraus 1966) associated with a good angular resolution. But the horn beam shape also determines the proportion of the beam that does not hit the mirrors, called "spillover", which makes a path for stray light to reach the receivers. Large effective apertures produce narrow beams on the sky and high levels of spillover. These are the terms of a trade-off between spillover and angular resolution for a given diameter of the telescope, a given wavelength, and a given shape of the horn beam pattern. This was a driver for the design of the satellite, the telescope and of both instruments. Optimizing the trade-off between resolution and rejection of stray light has driven us to the use of shaped and flared corrugated horns that can provide nearly Gaussian beams (Murphy et al. 2002; Maffei et al. 2010), which is the optimal solution. Corrugated horns are much better adapted to these requirements than the Winston cones most often used with bolometers (Baranov \& Mel'nikov 1966; Harper et al. 1976; Welford \& Winston 1978). The very large span of frequencies (one order of magnitude for HFI only) using a single $1.5 \mathrm{~m}$ telescope (Tauber et al. 2010b) required different solutions for the various parts of the spectrum.

The angular resolution of the $100 \mathrm{GHz}$ and $143 \mathrm{GHz}$ channels was degraded to 9.6 and $6.9^{\prime}$ respectively instead of the $5^{\prime}$ that the sensitivity of the bolometers would have allowed. The other channels were designed to define beams on the sky of about 5'. For the 217 and $353 \mathrm{GHz}$ channels, the resulting spillover and aperture efficiency are perfectly acceptable. But keeping the same beam size and the same design for the 545 and $857 \mathrm{GHz}$ channels would have produced aperture efficiencies of less than 0.1 and a corresponding loss of sensitivity to point sources. Reducing the beam size to keep the efficiency would have driven the whole scanning strategy of the satellite as well as the required data rate for reasons not directly related to the core of the mission. The final design of the two high frequency channels is based on horns able to accept several modes, 


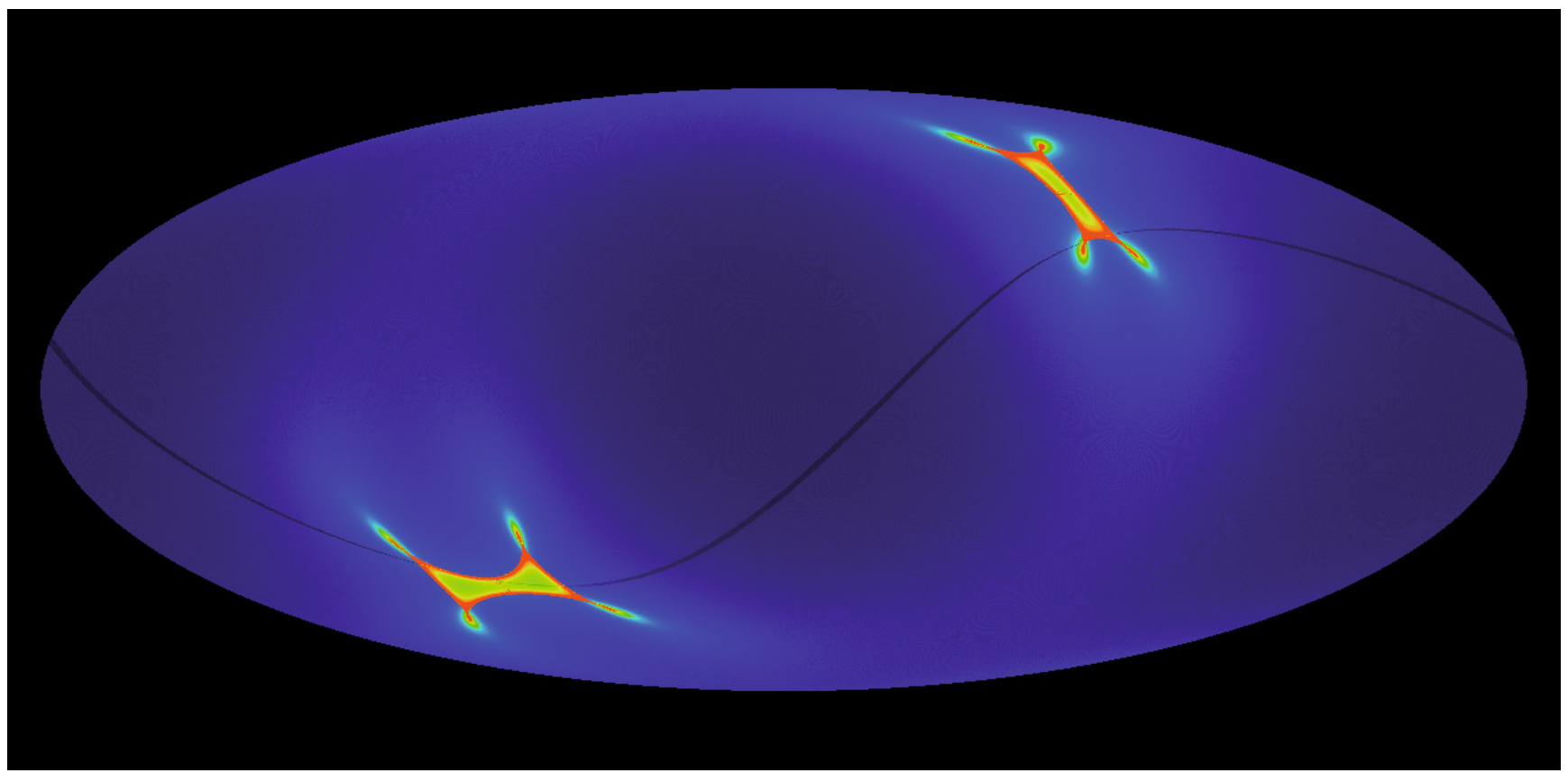

Fig. 3. Redundancy for one year of observation of a $353 \mathrm{GHz}$ receiver presented in the Galactic reference frame. Red: 5600 "hits" and dark blue: 300 "hits". Pixels are $2^{\prime}$ by $2^{\prime}$.

Table 2. Angular resolution and spillover (\%) of the single mode channels. Channels are identified in Fig. 4.

\begin{tabular}{lcccc}
\hline \hline Channel & $100-1$ & $143-1$ & $217-8$ & $353-5$ \\
\hline Angular resolution & 9.6 & 6.9 & 4.6 & 4.6 \\
Ideal spillover (\%) & 0.36 & 0.32 & 0.24 & 0.070 \\
Median measured spillover (\%) & 0.39 & 0.38 & 0.33 & 0.075 \\
\hline
\end{tabular}

giving both beams of about $5^{\prime}$ and high aperture efficiency. Their design was successful, although modelling of the whole optical chain proved to be a difficult exercise (Murphy et al. 2010). Experimental and theoretical work on these issues are on-going and will be reported at a later date. The first observations of planets with the multi-mode channels will produce more accurate information on the beam shape and the optical efficiency of these channels to point sources.

The resulting spillover is less than $0.5 \%$ in all channels (Table 2). Stray light is mainly produced either with zero or only one reflection on the mirrors (Maffei et al. 2010).

\subsection{Scanning strategy and redundancy}

A halo orbit around the L2 Lagrange point of the Sun-Earth system allows coverage of the full sky in about six months by rotating at one revolution per minute (RPM) about an axis nearly perpendicular to the Sun (Tauber et al. 2010a) and scanning the beams on the sky in nearly great circles (Dupac \& Tauber 2005). The baffle around the telescope and the orientation of the satellite minimize the contamination by the Sun, the Earth and the Moon.

Gaussian beams of $5^{\prime}$ FWHM are well-sampled with sampling intervals of $2^{\prime}$, which yields a detector sampling frequency of $180 \mathrm{~Hz}$. The $4 \pi$ solid angle contains about $3.7 \times 10^{7}$ independent samples separated by intervals of $2^{\prime}$. Redundancy is an essential ingredient of CMB measurements, because it provides a way to remove systematic effects and to perform null tests to verify the statistical properties of the data. Redundancy with such a scanning strategy is far from uniform (Table 3). Ecliptic polar regions are observed more frequently than the ecliptic equator and scanned in many more directions.

About thirty months is the longest survey duration allowed by the dilution cooler, estimated from tests at the Centre Spatial de Liège (CSL). The resulting number of independent observations of a given point of the sky will vary from 1000 to 60000 , depending on the band and the position on the sky (Fig. 3). The two ecliptic poles are observed more often than the other parts of the sky. The exact distribution depends on the chosen parameters for the scanning strategy and on the receiver. The de-pointing of the spin axis will be done by steps of two minutes of arc with a dwell time of 38 to $62 \mathrm{~min}$, following a cycloid-like modulation of the spin axis direction with a six months period that keeps the solar aspect angle constant. Scanning with a stable spin axis results in a quasi-periodic signal, providing a powerful means to measure the noise, test the instrument, identify and remove systematic effects.

The layout of the receivers (Fig. 4) is consistent with the scanning strategy (Ade et al. 2010). The HFI horns are positioned at the centre of the focal plane (Fig. 5), where the optical quality is good enough for the high frequencies. The curvature of rows compensates for the distortion of images by the telescope. A pair of identical SWB will scan the same circle on the sky to provide additional redundancy. Similar horns feeding PSBs are also aligned so that two pairs of PSBs rotated by $45^{\circ}$ with respect to each other scan the same line. This will provide the $Q$ and $U$ Stokes parameters with minimal correction for the pointing (Rosset et al. 2010). Residual systematics will come from the differences between the beam shapes of the two horns. In all cases except for the $100 \mathrm{GHz}$ horns, a measurement is also done by a pair of similar channels shifted by $1.25^{\prime}$ in the cross-scan direction, to ensure adequate sampling even in the worst case situations arising from uncertainties in pointing and in beam shapes. Every channel identified by a pair number is shifted towards the axis of rotation of the satellite. 
Table 3. Estimated average redundancy of observations in one year of operation for $2^{\prime} \times 2^{\prime}$ pixels. The average observation time is per bolometer.

\begin{tabular}{lccccccccc}
\hline \hline Frequency (GHz) and mode & $100 \mathrm{P}$ & $143 \mathrm{P}$ & 143 & $217 \mathrm{P}$ & 217 & $353 \mathrm{P}$ & 353 & 545 & 857 \\
\hline Average obs time (s/sample) & 2.9 & 1.8 & 1.8 & 0.85 & 0.85 & 0.85 & 0.85 & 0.85 & 0.85 \\
Average redundancy (1 year) & 1380 & 850 & 1250 & 400 & 600 & 400 & 600 & 600 & 600 \\
Max. redundancy (1 year) & 12000 & 7700 & 12000 & 3700 & 5600 & 3700 & 5600 & 5600 & 5600 \\
Min. redundancy (1year) & 700 & 420 & 630 & 200 & 300 & 200 & 300 & 300 & 300 \\
\hline
\end{tabular}

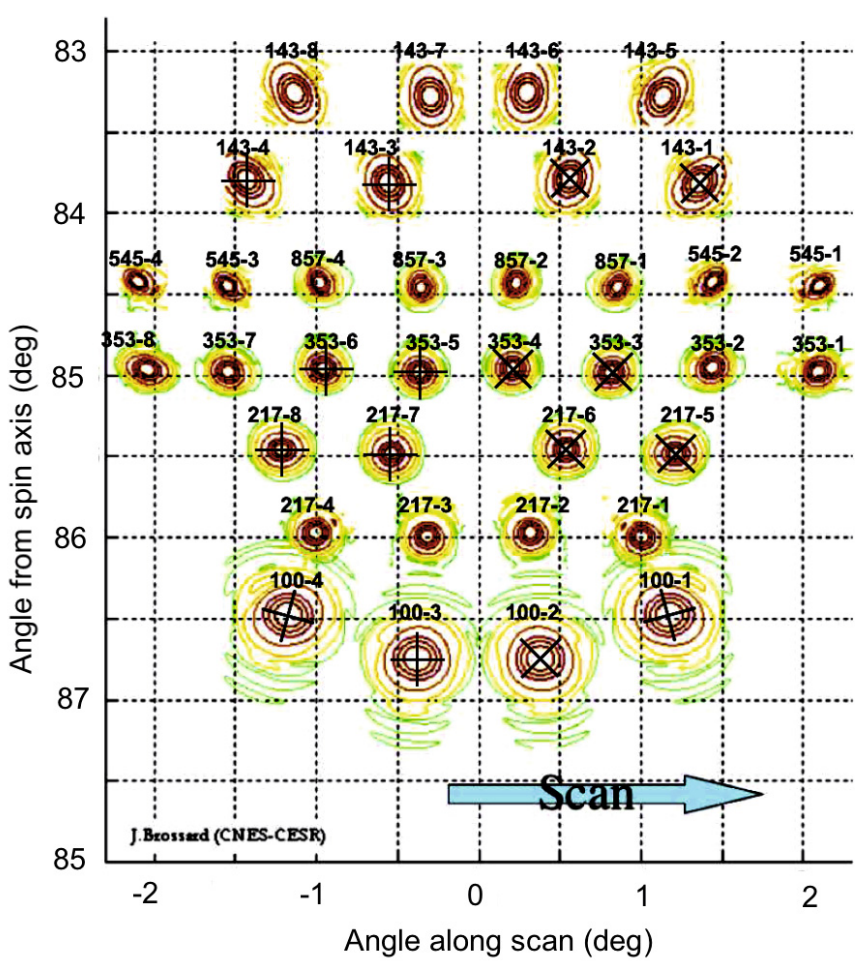

Fig. 4. Distribution of the beams on the sky and identification of optical channels (view from sky to telescope; units are degrees). Crosses indicate the polarisation orientation of the PSBs.

\section{Obtaining the desired performance}

\subsection{Transforming photons in digital data}

While the satellite rotates at one RPM, the image of the sky is convolved with the beam of the optical system, including the telescope, horn, internal optics, and the bolometers. Photons are also selected in frequency by the optical chains. This produces a timeline of optical power, which includes a nearly constant signal produced by the thermal emission of the telescope and the HFI cryogenic stages. The optical power (including the photon shot noise) is absorbed by the bolometers. The temperature changes of the bolometer are detected by current biasing of the bolometers and measuring the voltage at its output. Additional noise comes from the bolometer itself and from the readout electronics. The signal is then amplified, digitised, compressed and delivered to the telemetry system.

This results in a complex processing system involving a number of free parameters and variables (Fig. 6). Among them, the temperature of the $100 \mathrm{mK}$ stage is a critical variable, since it impacts directly on the temperature of the bolometers, changing their impedance, response to optical signals, noise and time response. The temperatures of the $4 \mathrm{~K}$ and $1.6 \mathrm{~K}$ stages directly drive the thermal emission of these stages. These effects were calibrated and the relevant temperatures are actively controlled

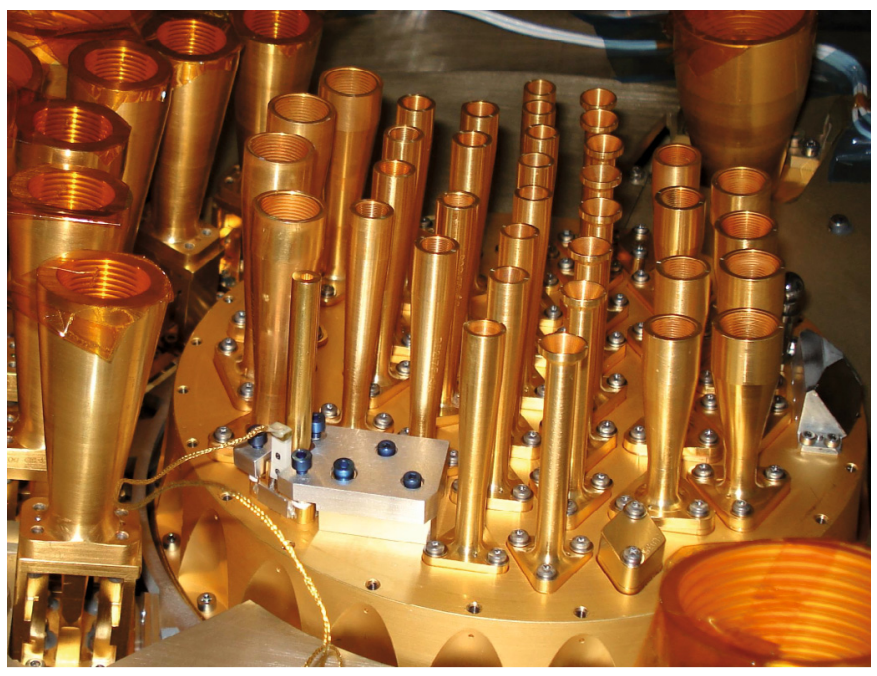

Fig. 5. One can see the HFI and the LFI horns on this picture taken during ground tests of the Proto-Flight Model. An additional horn, mounted for alignment tests, is visible at the end of the $100 \mathrm{GHz}$ row.

and residual fluctuations accurately measured so that their effects can be removed if necessary.

The parameters of the readout electronic unit (REU) are set by remote commands sent through the uplink to the spacecraft. Their effect on the bolometer response will be detailed in Sect. 4. Additional parasitic signals are expected from electromagnetic interferences, mechanical vibrations, and cosmic rays hitting various parts of the detectors.

\subsection{Time response - NEP trade-off}

Low temperature bolometers are the most sensitive receivers for wide band detection in the sub-THz frequency range. However, they have a finite time response set by their heat capacity and thermal conductivity. $\tau=C / G_{\text {eff }}$, where $C$ is the heat capacity of the bolometer and $G_{\text {eff }}$ is the effective thermal conductance (Chanin \& Torre 1984) of the heat link to the bolometer housing (the physical thermal conductance and its effective reduction because of electrothermal feedback). A bolometer time constant less than $1 / 3$ the time taken by a Gaussian beam to sweep across a given point on the sky will not significantly limit the signal bandwidth (Hanany et al. 1998). The beam size produced by the telescope and the optics, coupled with the scan rate $\left(6^{\circ}\right.$ per second), sets the maximum frequency range of the variations of the signal incident on the bolometers to a few tens of $\mathrm{Hz}$ (about $50 \mathrm{~Hz}$ for a $5^{\prime}$ beam). The time response of the HFI bolometers must be well-matched to this range, which is obtained for each bolometer species by tuning the thermal conductance $\mathrm{G}$.

The voltage response of the bolometer to incoming radiation depends on its temperature change for a given signal and is inversely proportional to the thermal conductance. The quantum 


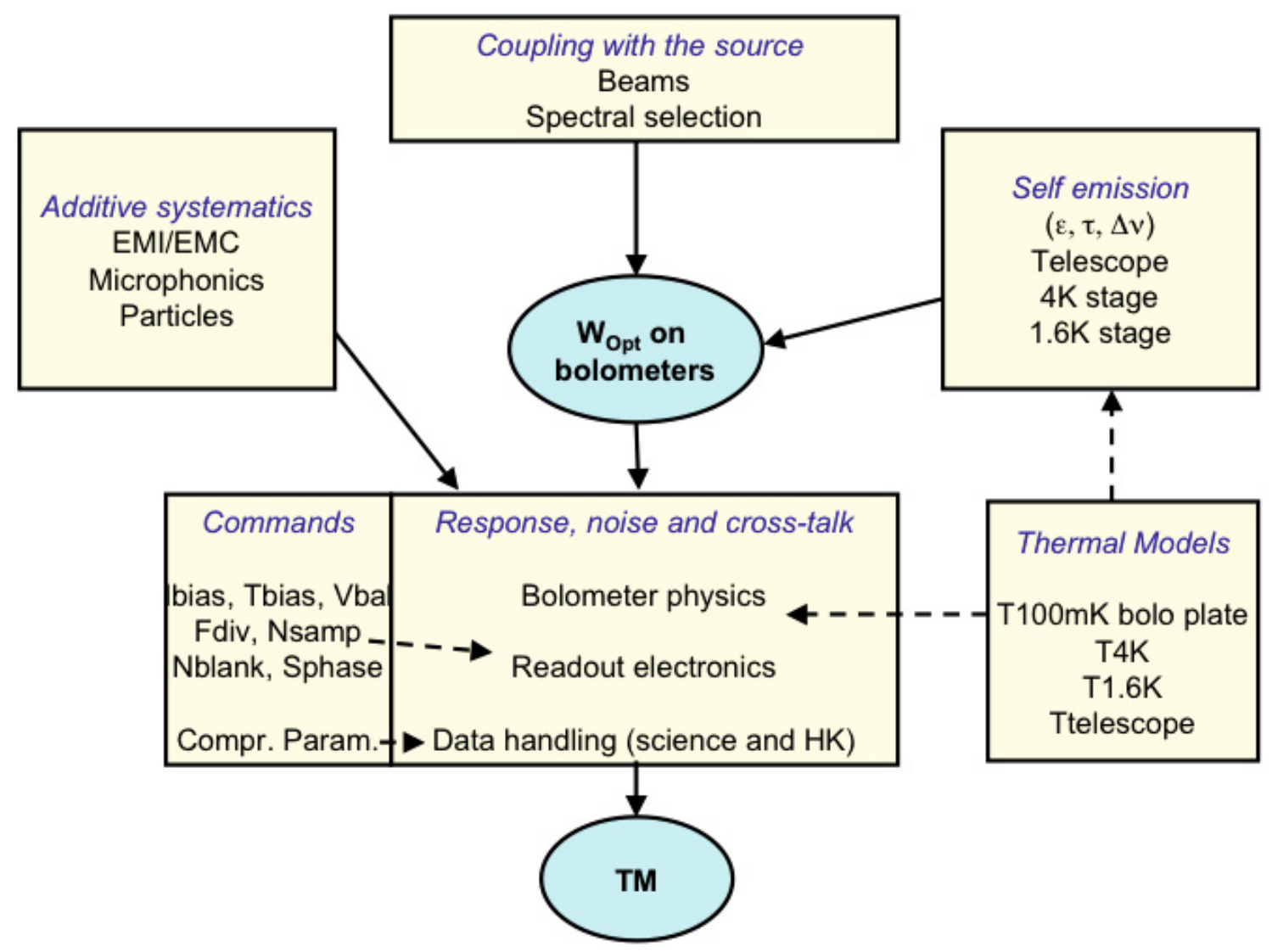

Fig. 6. Formation of the signal in HFI.

thermal fluctuations (phonon noise) is proportional only to the square root of thermal conductance. Lowering the conductance therefore decreases the NEP, but it also increases the time constant. The only way to obtain both a fast response and a low NEP is to reduce the heat capacity $C$, which can be done by optimizing the design of the bolometer and by cooling the device to very low temperatures. Most of the materials used for the bolometer fabrication exhibit a dramatic decrease in specific heat at very low temperatures. For the materials required to build the spider web bolometers (Bock et al. 1995), it was shown (Doucerain et al. 1995) that the requirements of HFI could be met only by cooling the bolometers to $100 \mathrm{mK}$. The choice of cooler temperature had to be made very early in the development of the instrument because nearly all critical subsystems depend on it, including the dilution cooler, the readout electronics, and the bolometers. The results of the calibration of the HFI indicate that the choice of a bolometer plate temperature of $100 \mathrm{mK}$ was correct, providing the required sensitivity, acceptable time responses, and a lifetime long enough to perform several independent sky surveys (see Sect. 5 on cryogenics).

\subsection{Spider web and polarisation-sensitive bolometers}

The details of the HFI bolometer build are given in Holmes et al. (2008); here we summarize the design considerations.

Bolometers consist of (i) an absorber that transforms the incoming radiation into heat; (ii) a thermometer that is thermally linked to the absorber and measures the temperature changes; and (iii) a weak thermal link to a thermal sink, to which the bolometer is attached.
In the spider-web bolometers, or SWBs (Bock et al. 1995; Mauskopf et al. 1997), the absorbers consist of metallic grids deposited on a $\mathrm{Si}_{3} \mathrm{~N}_{4}$ substrate in the shape of a spider web. The mesh design and the impedance of the metallic layer are adjusted to match vacuum impedance and maximise the absorption of millimetre waves, while minimising the cross section to particles. The absorber is designed to offer equal impedance to any linearly polarised radiation. Nevertheless, the thermometer and its electrical leads define a privileged orientation (Fig. 7) that makes the SWBs slightly sensitive to polarisation, as detailed in a companion paper (Rosset et al. 2010). The thermometers are made of neutron transmuted doped (NTD) germanium (Haller et al. 1996), chosen to have an impedance of about $10 \mathrm{M} \Omega$ at their operating temperature.

The absorber of PSBs is a rectangular grid (Fig. 7) with metallization in one direction (Jones et al. 2003). Electrical fields parallel to this direction develop currents and then deposit some power in the grid, while perpendicular electrical fields propagate through the grid without significant interaction. A second PSB perpendicular to the first one absorbs the other polarisation. Such a PSB pair measures two polarisations of radiation collected by the same horns and filtered by the same devices, which minimises the systematic effects: differences between polarised beams collected by a given horn are typically less than $-30 \mathrm{~dB}$ of the peak. The differences in the spectral responses of a PSB pair also proved to be a few percent in the worst case. Each pair of PSBs sharing the same horn is able to measure the intensity Stokes parameter and the $Q$ parameter associated with its local frame. An associated pair of PSBs rotated by $45^{\circ}$ scans exactly the same line (if the geometrical alignment is perfect), providing the $U$ Stokes parameter. 


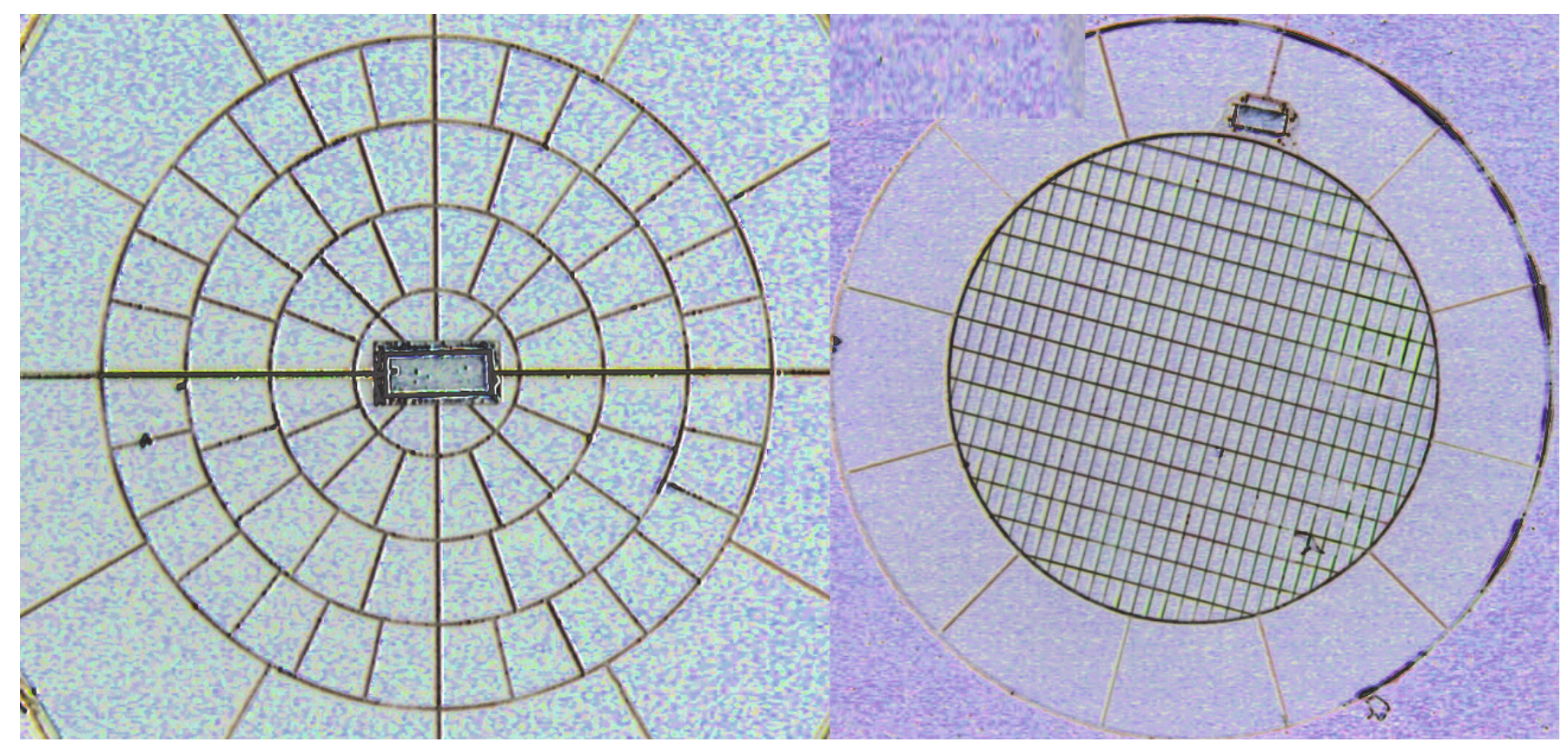

Fig. 7. Picture of a $143 \mathrm{GHz}$ spider web bolometer (left) and of a $217 \mathrm{GHz}$ polarisation-sensitive bolometer (right). One can see the temperature sensor at the centre of the SWB and at the upper edge of the PSB.

Table 4. The time response of each bolometer family is given by the average cut-off frequency of transfer functions.

\begin{tabular}{lccccccccc}
\hline \hline Channel & $100 \mathrm{P}$ & $143 \mathrm{P}$ & 143 & $217 \mathrm{P}$ & 217 & $353 \mathrm{P}$ & 353 & 545 & 857 \\
\hline Beam size (') & 9.6 & 6.9 & 7.1 & 4.6 & 4.6 & 4.6 & 4.7 & 4.7 & 4.3 \\
Average cut-off frequency @3dB $(\mathrm{Hz})$ & 14 & 29 & 28 & 26 & 55 & 27 & 52 & 74 & 72 \\
\hline
\end{tabular}

Notes. The suffix P stands for polarisation sensitive bolometers.

The PSBs define the overall polarisation sensitivity of the HFI. The two PSBs located in the same integrating cavity show a cross-polarisation leakage of several percent. Thus any PSB also shows a weak response to waves orthogonal to the polarisation it was designed for. Another non-ideal behaviour is that each PSB is positioned by hand in the bolometer housing with some alignment error. This makes the PSBs significantly different from ideal receivers. Nevertheless all the information needed to recover the polarisation of the incoming radiation is present in the acquired signal (Rosset et al. 2010).

The bolometers were developped by the Jet Propulsion Laboratory and Caltech (Holmes et al. 2008). They were extensively measured at $100 \mathrm{mK}$ and selected to provide the flight and spare bolometers. They were independently re-measured at Cardiff University. These measurements were used to assign each bolometer either to a particular location in the focal plane or as a spare. The selection was made to optimize the sensitivity across the frequency range needed for the measurement, $0.016 \mathrm{~Hz}$ to a few tens of $\mathrm{Hz}$, depending on the beam width. The average speed ( $3 \mathrm{~dB}$ cut-off frequency) achieved with the HFI bolometers is shown in Table 4. Parameters shown here are at settings of the readout electronics (particularly the bias current) that optimize the trade-off between NEP and signal bandwidth. Bolometers for the low frequency channels have to be larger to absorb efficiently incoming radiation. They thus have a large heat capacity and lower cut-off frequency. However, the larger beam size relaxes the bandwidth requirements. The average sensitivity for each family of bolometers is better than the requirements and even near to the goal, as shown in Sect. 3.6.

\subsection{Transfer function of the measurement chains}

The time response of bolometers is, in general, represented by a first order low pass filter (Mather 1982), due mainly to the thermal time constant modified by the electro-thermal feedback, i.e. the effect of electrical power deposited by the bias current. Some authors have considered the effect of capacitors in a direct current (DC) readout circuit, or the effect of distributed heat capacity in the bolometer (Vaillancourt 2005). The time response of the HFI detection chains is more complex than any of these cases because of the alternative current (AC) bias and the presence of an important stray capacitance (more than $100 \mathrm{pF}$ ) in the wiring between the bolometers and the JFET box (see Sect. 4 on readout electronics). The time response shows a steep cut-off above the modulation frequency. The various families of bolometers show significantly different responses in the frequency domain (Fig. 8).

In addition an excess in the bolometer response below a few $\mathrm{Hz}$ was identified during the HFI calibration. The amplitude of the excess response ranges from one per mil to a few percent and is particularly relevant given the strategy for the in-flight calibration, which is based on the CMB dipole that is measured at $16.7 \mathrm{mHz}$. The low frequency excess response (LFER) is attributed to parasitic heat capacitances caused by impurities weakly connected to the bolometers. A model based on this assumption represents the experimental data accurately. High quality data on LFER is not yet available for 20 out of the 52 bolometers. Strategies for the in-flight calibration of the LFER are based on the comparison of maps built from scans in opposite directions, on planets observations, on relative 

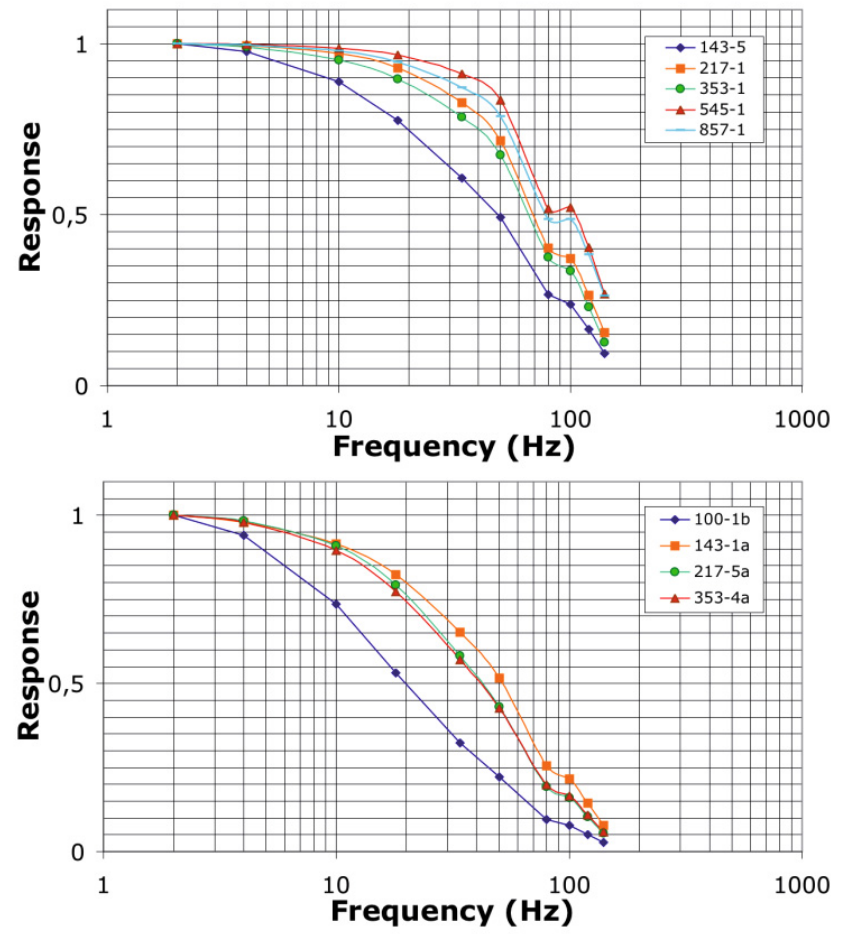

Fig. 8. Response in the frequency domain of one bolometer per family, normalized at $2 \mathrm{~Hz}$. Upper figure: unpolarized bolometers. Lower figure: polarized channels. The conditions are: optimal bias current, Fmod $=86 \mathrm{~Hz}$, base temperature $=102 \mathrm{mK}$, and nominal backgrounds .

calibration among different detectors, and on the response to steps in the bolometer bias current.

\subsection{Noise properties}

The sensitivity of all channels is limited mainly by fundamental sources of noise that have a Gaussian distribution and a white power spectral density: photon noise of the detected radiation, phonon noise (i.e. the quantum fluctuations of the phonon bath in the bolometers), and Johnson noise generated in the temperature sensor of the bolometer. Their spectrum is modified by the transfer functions of the various stages of the measurement chains.

The first stages of the amplification system, and especially the JFET stage, suffer from a low frequency noise that becomes significant below $10 \mathrm{~Hz}$. The AC bias moves the measured signal around the modulation frequency at $90 \mathrm{~Hz}$, in a domain well separated from the $1 / f$ noise, which can be filtered out before demodulation. This results in a remarkably flat noise spectrum, as shown in Sect. 4.

Additional sources of noise include thermal fluctuations from the cryogenic stages, voltages induced by vibration of the wires, heating of the $100 \mathrm{mK}$ plate induced by vibrations, and electromagnetic interference. We required that the rms value of each source of noise, or any source of loss in sensitivity, should be less than $30 \%$ of the photon noise by design, thus increasing the fundamental noise by less than $5 \%$. This was achieved in general, except for a few sources of noise, which are still significant, as described in the remainder of this section.

Two channels suffer currently from a random bi-stable noise known in pre-amplifiers as "telegraph noise". The number of affected channels varied after every disconnection and reconnection of the low temperature harness. During the test at CSL at system level, after which the harness was not moved, two channels, a $143 \mathrm{GHz}$ and a $545 \mathrm{GHz}$ SWB channel, were showing a significant level of telegraph noise, i.e. a level higher than half the standard deviation of the white noise. Algorithms for removing this source of noise were developped and are being tested on simulated signals.

Noise from external sources can also be observed. Glitches are due to cosmic rays entering the FPU through its metal box and heating various parts of the detection chain. The bolometers themselves and their associated structures can be heated by the energy from particles, which is always seen as positive peaks in the bolometer signal (Woodcraft et al. 2003; Tristram 2005). The decay time of these peaks depends on the thermal time constant of the heated item, ranging from a few milliseconds to $300 \mathrm{~ms}$ (Fig. 9). On the ground the rate of glitches did not exceed a few per hour, but up to several per minute are expected in flight. These glitches are most often above the noise and are easily detected and removed from the signal.

Electromagnetic interference and mechanical vibration (microphonics) effects were observed during the various stages of the tests. All but one of these effects could be attributed to the test facilities after analysis and correlation with the various thermometers, accelerometers and other sensors of the facilities. The remaining external noise is a strong parasitic signal produced at $40 \mathrm{~Hz}$ and harmonics by the compressors of the $4 \mathrm{~K}$ cooler, from both vibration and by electrical pick-up in the low noise part of the amplification chain. This effect had been anticipated, because it was considered unlikely that a periodic power of about 100 Watt in the compressors could be damped to less than $10^{-18} \mathrm{~W}$ at the level of bolometers. The compressor movement was phase-locked with the bias current and the sampling, which makes the parasitic lines extremely narrow. Interference occurs in the part of the circuit near to the bolometers with high impedance and low voltages where the signal is modulated by the AC bias. When demodulated in the readout electronics, the $40 \mathrm{~Hz}$ and harmonics produced by the $4 \mathrm{~K}$ cooler are folded at $10 \mathrm{~Hz}, 30 \mathrm{~Hz}$, etc. as can be seen in the noise spectrum shown in Fig. 10. Several methods were tested to remove them from the signal and proved to be extremely effective.

A two-component model provides a good fit to the noise spectra. The first component, arising from photon and phonon noise, is attenuated at high frequency by the bolometer time constant. The time-constant which is retrieved in the fit is compatible with the time constant of each bolometer. The second component, which characterizes the electrical noise (Johnson and electronics), is flatter. Even when observing a thermally unstable and non-uniform source in the ground-based facility, a simple decorrelation of the thermal background drift results in an improvement of the low frequency part of the power spectrum.

\subsection{Sensitivity}

The ground calibrations in Orsay provided us with a wealth of noise and response measurements in different environments. The noise equivalent delta temperature (NEDT) was evaluated in temperature and background conditions similar to those expected in flight. The NEDT is plotted in Fig. 11 for all bolometers and is compared with the requirements and goal sensitivity defined in Sect. 2.1.

The distribution of sensitivity of individual bolometers (Fig. 12) shows that about half the bolometers have a sensitivity better than the goal, but that the average is slightly higher than the goal. The main outliers are the $100 \mathrm{GHz}$ PSBs, which proved difficult to design and manufacture, and the $353 \mathrm{GHz}$ channels, 

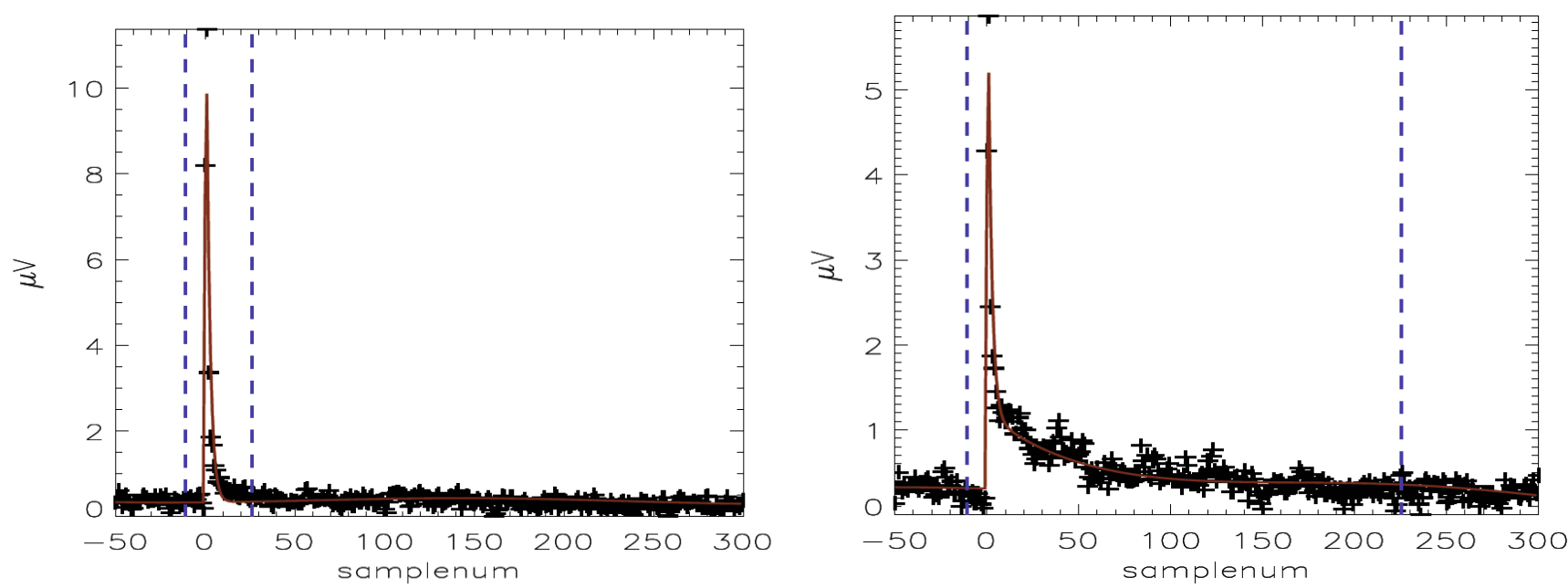

Fig. 9. Cosmic rays hitting the bolometers produce glitches with decay times depending on the part heated by the particle (after Tristram 2005).

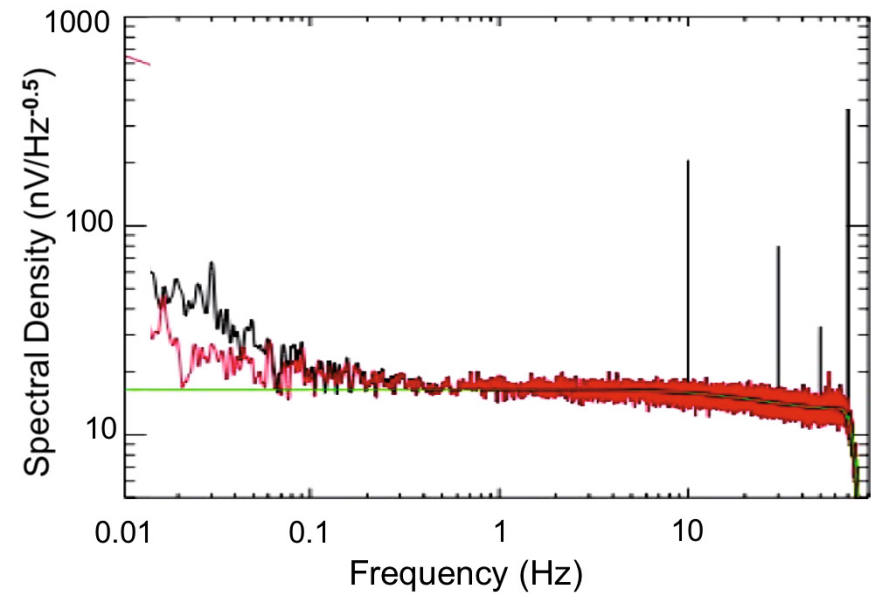

Fig. 10. Example of noise spectrum taken in the CSL facility. The black curve is a raw spectrum of three hours of data. The red curve is obtained by decorrelating the TOI in the time domain with a template made of other bolometer signals and smoothed. The green curve is a fit to the red power spectrum with a simple two-component noise model. The four high and narrow lines near 10, 30, 50 and $70 \mathrm{~Hz}$ are due to the $4 \mathrm{~K}$ coolers (see text).

because of unexpected difficulties in the manufacture of the long narrow horns imposed by the geometry of the focal plane.

We ignore the value of several parameters that will contribute to the noise during the mission, like the real environment conditions (particles and microphonics) or the effective thermal emission of the telescope. This emission will be determined partly from inevitable contamination by dust during the launch and probably from the degradation of the surface of the telescope by micro-meteorites during the mission. By extrapolating the results taken on the ground, one can derive estimates of the sensitivity (Table 5) that are very similar to the values published up to now (The Planck consortium 2005). Average sensitivity is for 14 months of operation for square pixels with the nominal beam size. The goal $\Delta T / T$ sensitivity (The Planck consortium 2005 ) is reported (in Italics) for comparison. Sensitivity to point sources assumes an $R-J$ spectrum and does not account for the confusion limit.

These results show that the bolometer programme was successful from the early design stages through to fabrication. The optical, thermal and electrical environment together with

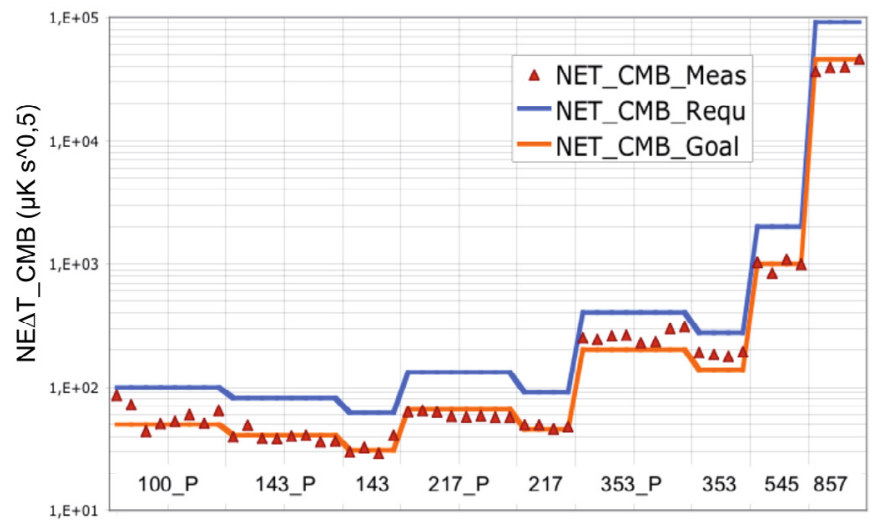

Fig. 11. Individual sensitivity of all bolometers measured during calibrations compared with requirements and goal.

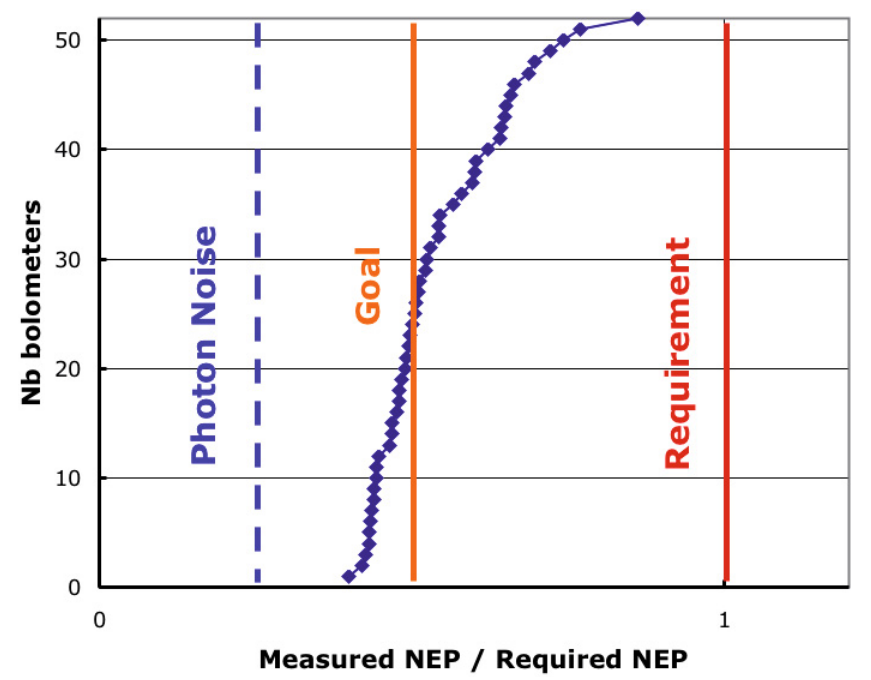

Fig. 12. Distribution of the sensitivity (linear scale) of HFI's 52 bolometers estimated from ground measurements and normalized by the sensitivity requirement. About half the bolometers should have a sensitivity better than the goal.

associated low-noise readout electronics were also designed and implemented successfully. They demonstrate that a complete system was developped although based on a new architecture 
Table 5. HFI performance expected from ground calibrations and for 14 months of operation.

\begin{tabular}{|c|c|c|c|c|c|c|c|c|c|}
\hline Channel & $100 \mathrm{P}$ & $143 \mathrm{P}$ & 143 & $217 \mathrm{P}$ & 217 & $353 \mathrm{P}$ & 353 & 545 & 857 \\
\hline Central frequency $(\mathrm{GHz})$ & 100 & 143 & 143 & 217 & 217 & 353 & 353 & 545 & 857 \\
\hline Bandwidth $(\%)$ & $33 \%$ & $32 \%$ & $30 \%$ & $29 \%$ & $33 \%$ & $29 \%$ & $28 \%$ & $31 \%$ & $30 \%$ \\
\hline Full width half maximum beam size $\left({ }^{\prime}\right)$ & 9.6 & 6.9 & 7.1 & 4.6 & 4.6 & 4.6 & 4.7 & 4.7 & 4.3 \\
\hline Number of detectors & 8 & 8 & 4 & 8 & 4 & 8 & 4 & 4 & 4 \\
\hline$(\Delta T / T)_{\mathrm{CMB}}$ per pixel $(\mu \mathrm{K} / \mathrm{K}, I$ Stokes parameter $)$ & 3 & \multicolumn{2}{|c|}{2.2} & \multicolumn{2}{|c|}{4.8} & \multicolumn{2}{|c|}{20} & 150 & 6000 \\
\hline Proposal goal ( $\mu \mathrm{K} / \mathrm{K}$, I Stokes parameter $)$ & 2.5 & \multicolumn{2}{|c|}{2.2} & \multicolumn{2}{|c|}{4.8} & \multicolumn{2}{|c|}{14.7} & 147 & 6700 \\
\hline$(\Delta T / T)_{\mathrm{CMB}}$ per pixel $(\mu \mathrm{K} / \mathrm{K}, Q$ and $U$ Stokes parameters $)$ & 4.8 & \multicolumn{2}{|c|}{4.1} & \multicolumn{2}{|c|}{9} & \multicolumn{2}{|c|}{38} & - & - \\
\hline Proposal goal $(\mu \mathrm{K} / \mathrm{K}, Q$ and $U$ Stokes parameters $)$ & 4.0 & \multicolumn{2}{|c|}{4.2} & \multicolumn{2}{|c|}{9.8} & \multicolumn{2}{|c|}{29.8} & _ & _ \\
\hline Sensitivity to point sources (mJy) & 14 & \multicolumn{2}{|c|}{10} & \multicolumn{2}{|c|}{12} & \multicolumn{2}{|c|}{30} & 40 & 35 \\
\hline
\end{tabular}

and new components and meets the most optimistic expectations in the early stages of the Planck project.

\section{Readout electronics and on-board data handling}

\subsection{Principles of operation of the readout electronics}

The performance of the bolometers cannot be separated from the performance of the readout electronics used to measure the impedance of the thermometer part of the bolometer. The bias current deposits some power in the bolometer and changes its temperature. AC biased readout electronics developped in the late 80s (Rieke et al. 1989) have the advantage of producing a noise spectrum that is flat down to very low frequencies, while DC biased readouts show a large $1 / f$ component at frequencies less than about $10 \mathrm{~Hz}$. The AC bias readout electronics of the HFI instrument (Gaertner et al. 1997) includes a number of original features proposed by several laboratories (CRTBT in Grenoble, CESR in Toulouse and IAS in Orsay), which were validated on the Diabolo experiment and on the balloon-borne Archeops experiment. It was developped for space by the CESR in Toulouse.

The particular features of the HFI AC bias readout are mainly (i) that the cold load resistors were replaced by capacitors because they have no Johnson noise; (ii) that the detectors are biased by applying a triangular voltage to the load capacitors which produces a square current $\left[I_{\text {bias }}\right]$ in the capacitors, and a square voltage $\left[T_{\text {bias }}\right.$ ] that compensates for the stray capacitance of the wiring (producing a nearly square bias current into the bolometer, as shown in Fig. 13); (iii) that a square offset compensation signal is subtracted to the bolometric signal to minimise the amplitude of the signal that has to be amplified and digitized; (iv) that the electronic scheme is symmetrical and uses a differential amplification scheme to optimize the immunity to electromagnetic interferences; (v) and finally that every parameter of the REU (listed below) can be set by commands, which is made possible by using digital-to-analog and analog-to-digital converters extensively.

The modulation frequency $f_{\text {mod }}$ of the square bias current can be tuned from $70 \mathrm{~Hz}$ to $112 \mathrm{~Hz}$ by the telecommand parameters $N_{\text {sample, }}$, which defines the number of samples per half period of modulated signal, and by $f_{\text {div }}$ which determines the sampling frequency of the ADC. The optimal frequencies are around $90 \mathrm{~Hz}$.

Each channel has its own settings:

$I_{\text {bias }}$ amplitude of the triangular bias voltage;

$T_{\text {bias }}$ amplitude of the transient bias voltage;

$V_{\text {bal }}$ amplitude of the square compensation voltage;
$G_{\text {amp }}$ value of the programmable gain of the REU [1/3, 1 , $3,7.6]$;

$N_{\text {blank }}$ number of blanked samples at the beginning of halfperiod not taken into account during integration of the signal; $S_{\text {phase }}$ shift when computing the integrated signal.

All these parameters influence the effective response of the detection chains, and were optimized during the calibration campaigns. They will be tuned again during the calibration and performance verification (CPV) phase following the launch of Planck. The scientific signal is provided by the integral of the signal on each half-period, between limits fixed by the $S_{\text {phase }}$ and $N_{\text {blank }}$ parameters.

The interaction of modulated readout electronics with semiconductor bolometers is rather different from that of a classical DC bias readout (Jones 1953). The differences were seen during the calibration of the HFI, although the readout electronics was designed (Gaertner et al. 1997) to mimic the operation of a DC biased bolometric system as far as possible. With the $\mathrm{AC}$ readout the maximum of responsivity is lower and is obtained for higher bias current in the bolometer with respect to the DC model. This is caused by the stray capacitance in the wiring which has negligible effects for a DC bias and a major effect for an AC bias. In our case, a stray capacitance of $150 \mathrm{pF}$ induces increases of NEP ranging from $4 \%(857 \mathrm{GHz}$ bolometers) to $10 \%$ (100 GHz bolometers) and also affects the HFI time response. Models were developped and will be published in a forthcoming paper.

\subsection{Overview}

The readout electronics consist of 72 channels designed to perform low noise measurements of the impedance of 52 bolometers, two blind bolometers, 16 accurate low temperature thermometers, all in the $10 \mathrm{M} \Omega$ range, one resistor of $10 \mathrm{M} \Omega$ and one capacitor of $100 \mathrm{pF}$. The semiconductor bolometers and thermometers of Planck-HFI operate at cryogenic temperature around $100 \mathrm{mK}$ on the focal plane, with impedance of about $10 \mathrm{M} \Omega$ when biased at the optimal current. The readout electronics on the contrary have to operate at "room" temperature $(300 \mathrm{~K})$. The distance between the two extremities of the readout chain is about $10 \mathrm{~m}$ and could represent a point of extreme susceptibility to electromagnetic interference. The readout electronic chain was split into three boxes. These are the JFET box, located on the $50 \mathrm{~K}$ stage of the satellite at $2.2 \mathrm{~m}$ from the focal plane, the pre-amplifier unit (PAU), located $1.8 \mathrm{~m}$ further at $300 \mathrm{~K}$, and the REU (Fig. 14), located on the opposite side of the satellite, $5 \mathrm{~m}$ away. Each of the three boxes (JFET, PAU and REU) consists of 12 belts of six channels. The first nine belts 


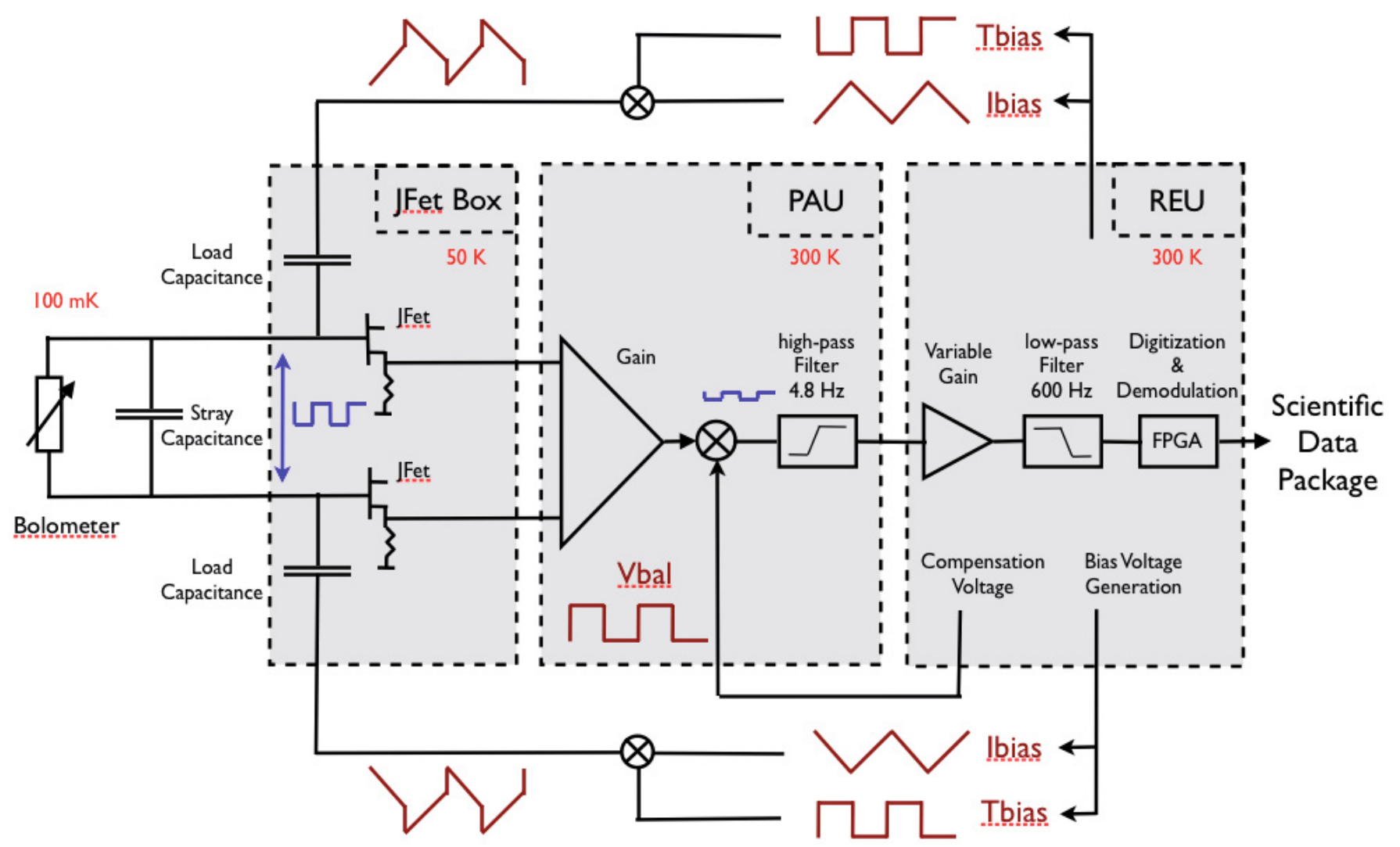

Fig. 13. Principles of the readout electronics.

are dedicated to the bolometers, and the three last ones to the accurate thermometers, the resistor and the capacitor.

\subsubsection{The JFET box}

The HFI semiconductor bolometers have resistance in the $10 \mathrm{M} \Omega$ range and operate at cryogenic temperatures, while most of the readout electronics must operate close to room temperature. As described above, the physical distance between the detectors and the room temperature electronics is a few metres. A cable this long at high impedance would be very sensitive to electromagnetic pickup and microphonics. It is therefore essential that the impedance of the signal is lowered as close as possible to that of the detectors. In our system this is accomplished by means of JFET source followers, located on the $50 \mathrm{~K}$ stage. There are two JFETs per channel, since the readout is fully differential, and they provide a current amplification of the signal while keeping the voltage amplification very close to unity. We selected low noise small-signal JFETs, with intermediate capacitance (process NJ450 from Interfet). The $50 \mathrm{~K}$ cryogenic stage can withstand a limited power load, and a maximum of $270 \mathrm{~mW}$ was reserved for the 144 JFETs. However, the noise of the JFETs decreases with increasing source current, so we had to find a trade-off between power dissipation and noise. Moreover, the noise of the JFETs decreases when lowering the temperature below room temperature, but JFETs cease to function at temperatures lower than $100 \mathrm{~K}$.

The JFET box (Brienza et al. 2006) is thermally linked to the back of the telescope at about $50 \mathrm{~K}$. Inside the box, the JFETs are mounted on a thermally insulated plate with an active temperature control to keep them at the optimal temperature of $110 \mathrm{~K}$. A picture of one module, without the super insulation blanket is shown in Fig. 15. With a dissipated power lower than $240 \mathrm{~mW}$, mainly produced by the JFETs and the source resistors, we obtained a noise power spectral density of less than $3 \mathrm{nV} \mathrm{Hz}^{1 / 2}$ for the frequency range of interest. This increases the total noise of all bolometer channels by less than $5 \%$.

\subsubsection{The preamplifier and readout electronics units}

The PAU is located as close as possible to the focal plane and provides an amplification of the low level voltages by a factor of 1000. The REU provides a further variable gain amplification (1 to 22.8) and contains all the interfaces between the ana$\log$ and the digital electronics. The bolometric signal is filtered by a second order high-pass filter at $4.8 \mathrm{~Hz}$, first in the PAU, and then by a second order low-pass filter at $600 \mathrm{~Hz}$ in the REU. The six channels on each of the 12 belts have their own power supply and are separated by solid shields limiting interference (effectively independent Faraday cages). Moreover each mechanic part of the electronics was assembled with EMI/EMC seals, ensuring high immunity against interference.

Furthermore the nominal and redundant REU processors, located on the REU bloc with the 12 other belts of the chain, communicate with the data processing unit (DPU) by sending science and housekeeping data on one side, and with the pre-processors (FPGA) of each belt on the other side. The design of distributed power was adopted here to reduce the computing charge of the main REU processors by distributing a part of the calculation to the pre-processors of each belt. Thus the digitization was introduced early in the readout chain, with each belt working independently and performing a digitized demodulation. 

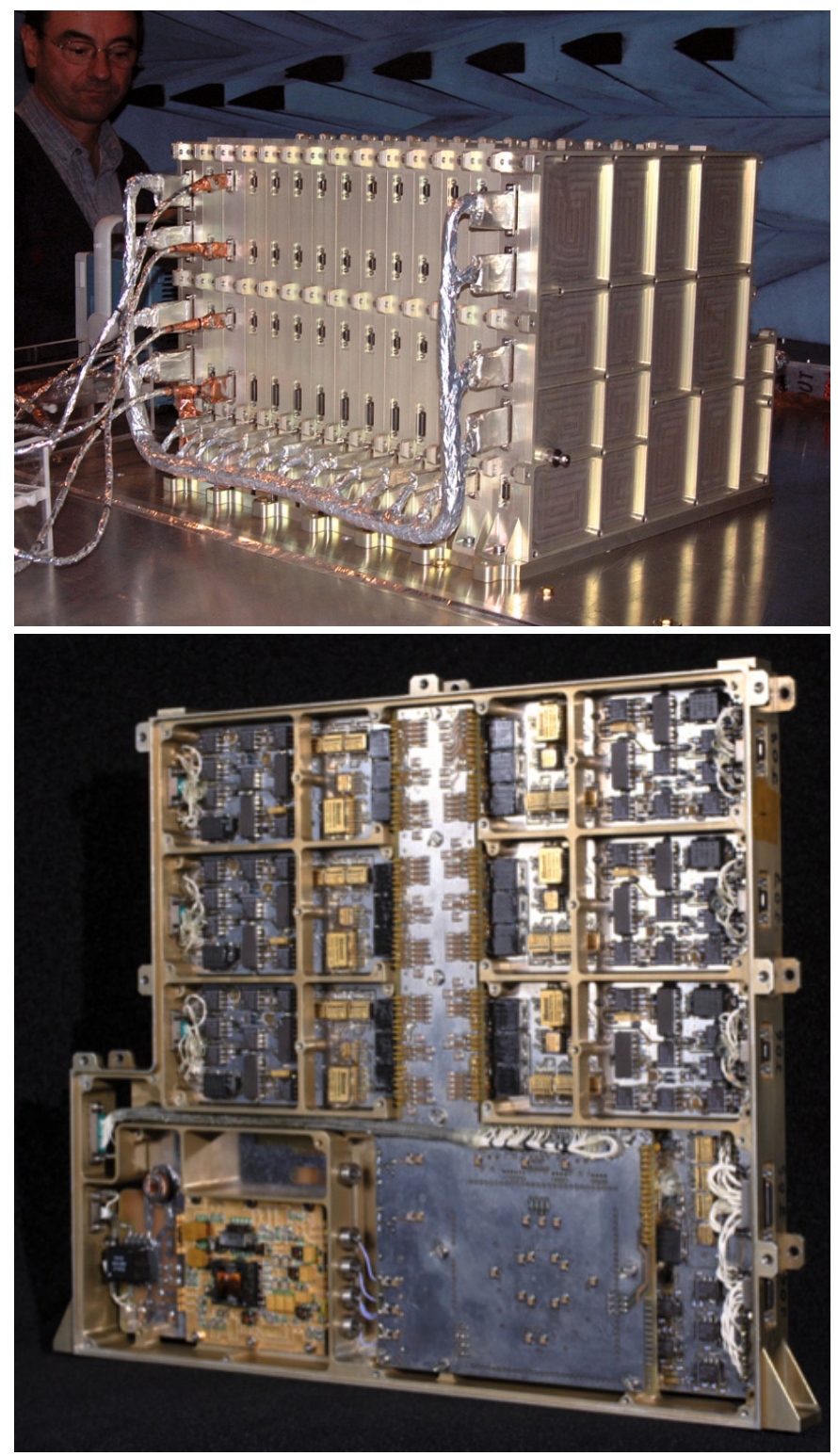

Fig. 14. The REU (top) consists of 12 belts (bottom) of six channels each.

The PAU \& REU electronics are made of about 58000 electronic components, with a total mass of $46 \mathrm{~kg}$ and a power supply of only $88 \mathrm{~W}$.

\subsubsection{Performance of the electronics}

The HFI bolometers provide an electrical signal in the $\mathrm{nV}$ range under impedance of about $10 \mathrm{M} \Omega$. Any pick-up from external signals can heat the bolometers and add noise (Yvon et al. 2008). An early analysis showed that it was essential to design the hardware with a high level of EMI immunity. This design was difficult because of the need for thermal isolation between warm and cold parts. Thus nanoVolt signals have to travel across more than seven metres before significant voltage amplification takes place in PAU. This was achieved by designing the FPU as a closed box and by using EM gaskets, special shielded wires and harnesses. To ensure continuity of the Faraday cage in between the $4 \mathrm{~K}$ stage, $18 \mathrm{~K}$ stage, JFET box (located at $50 \mathrm{~K}$ on the frame of the telescope baffle) and the PAU on the service module (SVM),

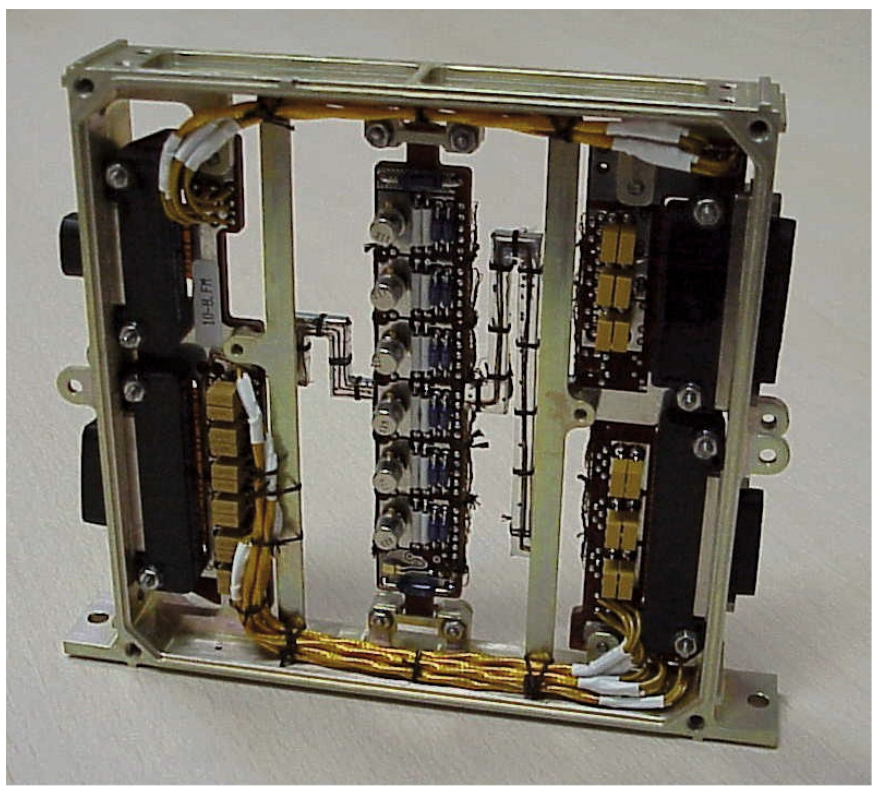

Fig. 15. Flight model of a six-channel JFETs board mounted on the $50 \mathrm{~K}$ stage. (Photo courtesy of Galileo Avionica.)

stainless steel bellows were used as a shield containing all the wires and harnesses.

The harness between the PAU and REU units represents another highly critical section of the readout chain sensitive to EMI/EMC. Double shielding with aluminium and frequent grounding of the harness provide a susceptibility of $5 \mathrm{~V} / \mathrm{m}$ as required for space equipment. In addition, a numerical simulation of the EMI susceptibility of the system and its grounding scheme was developped. As simulations predicted, the careful shielding described above was not satisfactory on its own: ground currents flowing in the mechanical structure and in shields contaminated the readout through capacitive coupling with the high impedance readout lines. We had to use materials (mainly Sapphire) showing excellent thermal conduction and electrical isolation properties to open contamination paths at critical places in the mechanical structure surrounding the HFI instrument. This design was validated by a series of specific tests.

During the EMC tests performed in the Toulouse INTESPACE EMC laboratory, the conducted and radiated emission as well as the susceptibility of the full readout electronics were measured and found to match all spacecraft specifications. The system test at satellite level performed at CSL confirmed the validity of the design. Nevertheless, as anticipated, we found lines on the scientific signal produced by the periodic current driving the compressors of the $4 \mathrm{~K}$ cooler. These lines can be easily removed from the signal (see the description of anomalous noise and systematic effects in Sect. 3). The design of the electronics also provided a low level of cross-talk between channels, with rejection ratios of typically $-70 \mathrm{~dB}$ for the JFET box and $-110 \mathrm{~dB}$ for the PAU \& REU. This was confirmed by tests at instrument level. The thermal stability of all critical components was chosen to provide a stability of the gains with temperature better than $80 \mathrm{ppm} / \mathrm{K}$. With other refinements of the design that will be reported in a future paper, this thermal stability was essential to ensure readout electronics free of low frequency noise down to $0.016 \mathrm{~Hz}$, which is the frequency at which the CMB dipole is measured (Fig. 16). 


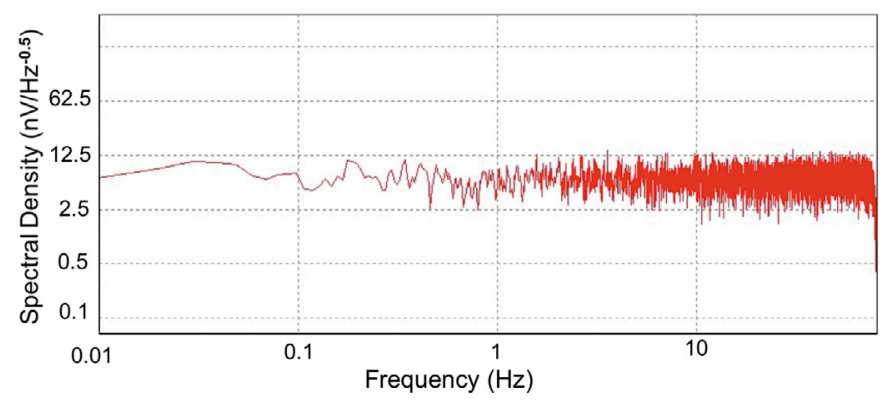

Fig. 16. Noise spectral density of the FM readout electronics. The level of $5.5 \mathrm{nV} \mathrm{Hz}^{-0.5}$ is obtained over the range $0.01 \mathrm{~Hz}$ to $600 \mathrm{~Hz}$.

\subsection{The data processing unit and data compression}

The DPU is the on-board computer of the HFI. It communicates with the satellite central computer (CDMU) through a MIL-1553 bus, and to the REU, the $4 \mathrm{~K}$ cooler and dilution cooler electronics through specifically designed lines. It is built around a TEMIC 21020 digital signal processor (DSP) running under the real time operating system "Virtuoso". All sensitive components (processor, memories, FPGAs) are radiation hardened, which highly reduces the risk of a breakdown induced by cosmic rays. A cold redundant unit provides an extra gain in reliability. The DPU was developped by LAL at Orsay.

A set of telecommands (TC) permits the configuration of the operating mode and every sub-system. The housekeeping (HSK) data flow $\left(4 \mathrm{kbit} \mathrm{s}^{-1}\right)$ gives the status of the main parameters every second and the most recent value of all available parameters every minute. If needed, a new version of the application software (ASW) can be uploaded through specific TCs.

The science data flow is limited by the CDMU memory allocation to $75 \mathrm{kbit} \mathrm{s}^{-1}$, which is obtained mainly by a compression algorithm based on the transmission of differences between neighbouring data. The required compression factor is finally obtained through the tuning of the quantization step $q$, uploaded channel by channel through TCs. In the standard operation mode, its value is half the white noise rms $\left(\sigma_{\text {White }}\right)$ of the channel, which results in an increase of the noise of about $1 \%$.

From a more technical point of view, data from the REU are time-ordered channel per channel in a local memory buffer and gathered in a "compression slice" of 254 consecutive data samples of a single channel. During the same period, data of the previous compression slice are processed and compressed. A mean value is computed and downloaded and the difference between every sample and the mean value is coded in $q$ step units and downloaded into a science data packet in a manner that optimizes the data flow. This algorithm was validated on simulated data based on the Planck Sky Model.

Under-sampling may provide a further method to reduce science data flow. This is implemented by downloading the mean value of the signal on every compression slice and only one sample every $n, 2<n<15$. Under-sampling leads to data loss unless only low frequencies are present in the signal. It can be applied only on some thermometer channels.

Finally, if the actual data rate exceeds the allocation (including a $10 \%$ margin), the amount of science data is limited for each ring of observations defined by the scanning strategy to distribute data losses evenly on the sky. This is expected to be a contingency mode, only triggered in exceptional circumstances, for instance during magnetic storms that can provoke bursts of glitches in the bolometer signal.

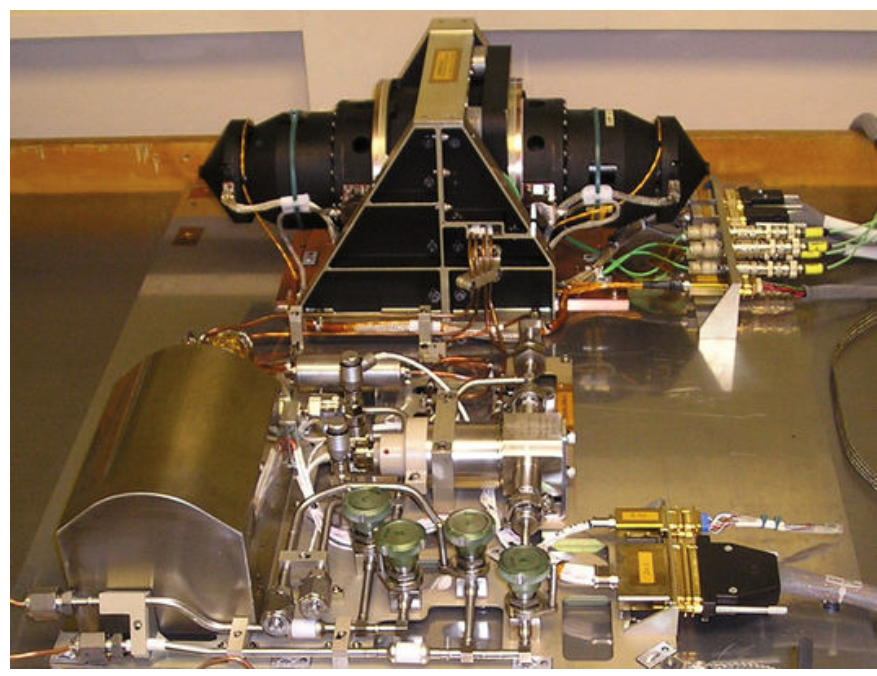

Fig. 17. The $4 \mathrm{~K}$ cooler compressors and gas cleaning equipment prior to integration in the spacecraft.

\section{Cryogenics and thermal design}

\subsection{Thermal requirements for photon noise limited photometry}

The sources of parasitic radiation are (i) the telescope, its baffle and all objects inside the baffle cavity, including the focal plane units of LFI and HFI; and (ii) the elements of the optical chain ensuring the coupling between the bolometers and the telescope. The parasitic radiation induces two kinds of noise: photon noise and the power background variations due to the temperature fluctuations of stray radiation sources.

The overall design of Planck is driven by the need to reduce parasitic radiation of thermal origin. Its thermal architecture is devided into two parts. The first part is always exposed to the Sun, and its temperature is therefore suitable for the operation of standard electronics and mechanisms. At the other end, the second part is always protected from the Sun and provides a cold environment for the telescope and for the cryogenically cooled focal plane instruments. This passive cooling architecture provides the radiative environment for the operation of three active coolers environment required by the receivers of LFI and HFI. The Sorption cooler (described in Tauber et al. 2010b) that cools the LFI (Bersanelli et al. 2010) at less than $20 \mathrm{~K}$ provides the pre-cooling stage needed by the $4 \mathrm{~K}$ and the coolers described in the next sections.

The noise produced by thermal fluctuations of stray radiation sources should ideally be small compared to the unavoidable photon noise. The criterion for all non-fundamental sources of noise (see Sect. 2 on noise budget) sets the required temperature stability of the cryogenic stages (Lamarre et al. 2003). The maximum spectral density of the temperature fluctuations in the useful frequency range $[16 \mathrm{mHz} ; 100 \mathrm{~Hz}]$ is specified below:

$4 \mathrm{~K}$ horns and filters: $10 \mu \mathrm{K} \mathrm{Hz}^{-0.5}$ (30\% emissivity)

$1.6 \mathrm{~K}$ filters: $28 \mu \mathrm{K} \mathrm{Hz}^{-0.5}$ (20\% emissivity)

$0.1 \mathrm{~K}$ bolometers plate: $20 \mathrm{nK} \mathrm{Hz}^{-0.5}$.

\subsection{The $4 \mathrm{~K}$ cooler}

The $4 \mathrm{~K}$ cooler is based on a helium closed-circuit Joule-Thomson (JT) expansion driven by two mechanical 


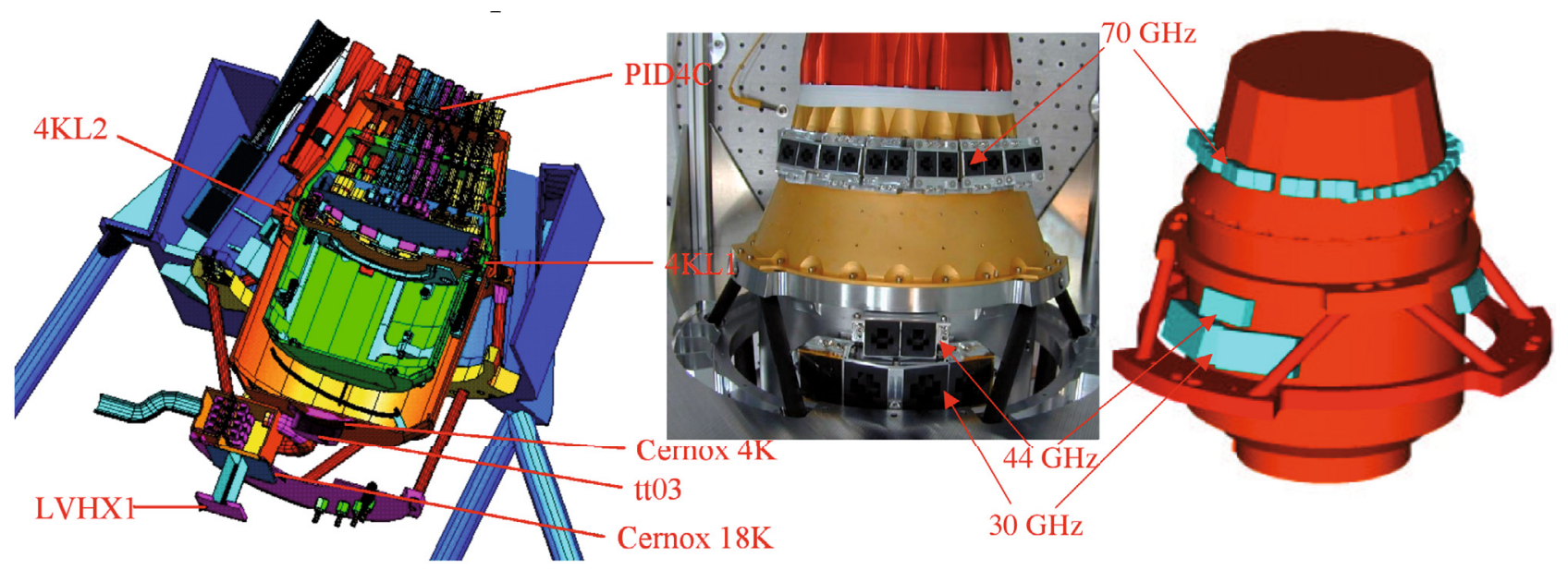

Fig. 18. Position of thermometers (left) and of the reference loads for the LFI (centre and right).

compressors in series. A description of this system is given in Bradshaw \& Orlowska (1997, p. 465). It was developped at RAL. The compressors for the HFI $4 \mathrm{~K}$ cooler were supplied by EADS Astrium in Stevenage, UK. The drive electronics were designed and built by a consortium of RAL and Systems Engineering and Assessment (SEA) in Bristol. The pre-charge regulator was built by CRISA in Madrid with supervision from the University of Granada.

The two compressors are mounted in symmetrical positions as shown in Fig. 17 first and foremost to cancel momentum transfer to the spacecraft. Furthermore, force transducers between the two compressors provide an error signal that is processed by the drive electronics servo system, which controls the profile of the piston motions to minimise the first seven harmonics of the periodic vibration injected into the spacecraft.

The $4 \mathrm{~K}$ cold stage is a small liquid helium reservoir, where the helium is contained in a sinter material. This is located on the $4 \mathrm{~K}$ stage after the expansion of the gas through the JT orifice (JT). This is an important point, as the JT orifice is thermally isolated from the stage. It is attached to the bottom of the $4 \mathrm{~K}$ box of the HFI focal plane unit (FPU) as can be seen in Fig. 19. It provides cooling for the $4 \mathrm{~K}$ shield and also pre-cooling for the gas in the dilution cooler pipes described in the next section.

The cooling power and the thermal properties of this cooler were measured by the RAL team and are summarised in the equation below, which gives its linear dependence on the main parameters in the vicinity of the flight operating point. These parameters are the pre-cooling temperature $T_{\mathrm{pc}}$, the $\mathrm{V}$-groove 3 temperature $T_{\mathrm{vg} 3}$, the stroke amplitude of the compressors $S_{\mathrm{a}}$ and the helium filling pressure $P_{\text {fill }}$.

$$
\begin{aligned}
& \text { Heat lift }=18.3 \mathrm{~mW}+3.4 \mathrm{~mW} / \mathrm{mm} \times\left(S_{\mathrm{a}}-7.5 \mathrm{~mm}\right) \\
& -1.1 \mathrm{~mW} / \mathrm{K} \times\left(T_{\mathrm{pc}}-17 \mathrm{~K}\right) \\
& +0.6 \mathrm{~mW} / \mathrm{bar} \times\left(P_{\text {fill }}-4.5 \mathrm{bars}\right) \\
& \text { Heat load }=10.6 \mathrm{~mW}+0.5 \mathrm{~mW} / \mathrm{K} \times\left(T_{\mathrm{pc}}-17 \mathrm{~K}\right) \\
& +0.065 \mathrm{~mW} / \mathrm{K} \times\left(T_{\mathrm{vg} 3}-45 \mathrm{~K}\right)+\text { Heater power } \\
& \begin{aligned}
T=4.47 \mathrm{~K}- & 0.12 \mathrm{~K} / \mathrm{mm} \times\left(S_{\mathrm{a}}-7.5 \mathrm{~mm}\right) \\
+ & 0.007 \times\left(T_{\mathrm{pc}}-17 \mathrm{~K}\right) \\
- & 0.032 \mathrm{~K} / \mathrm{mW} \times(\text { Heat lift }- \text { Heat load }) .
\end{aligned}
\end{aligned}
$$

The stroke amplitude and to some degree the temperature of the sorption cooler are adjustable in flight by telecommand. The $4 \mathrm{~K}$ cooling power margin strongly depends on the stroke amplitude, as the heat load increases and the heat lift decreases with the sorption cooler pre-cooling temperature. The temperature of the sorption cooler is thus the most critical interface of the HFI cryogenic chain. It is mostly driven by the warm radiator on the satellite, which will be at $272 \mathrm{~K}( \pm 10 \mathrm{~K}$, maximum range allowed), leading to sorption cooler temperatures between 16.5 $\mathrm{K}$ to $17.5 \mathrm{~K}$ (Bersanelli et al. 2010). The warm radiator temperature is also a critical interface.

A thermal balance system test showed that the pre-cooling temperature will be close to $17.5 \mathrm{~K}$. The performance given above indicates that the $4 \mathrm{~K}$ cooling power margin is about $5.2 \mathrm{~mW}$ for a stroke amplitude of $7 \mathrm{~mm}$, which is well below the maximum value of $8.8 \mathrm{~mm}$. In these conditions the temperature is about $4.4 \mathrm{~K}$, well below the maximum of about $4.7 \mathrm{~K}$ required for the operation of the dilution cooler with reasonable margins.

The two mechanical compressors produce micro-vibrations and also induce electromagnetic interference affecting the science signals of bolometers. The risks associated with these effects were taken into account early in the design of the HFI by phase-locking the sample frequency of the data to a harmonic of the compressors' frequency. No microphonic noise was seen in system tests when the vibration control option was activated in the drive electronics of the compressors. However, electromagnetic interferences were seen in the qualification and the flight model system tests at several beat frequencies of the compressor frequency and sampling frequency. But they are extremely narrow and can be removed completely from the signal as a result of the harmonic ratio between them.

Vibration from the compressors could affect the HFI data in a different way. During the instrument and system tests, the $100 \mathrm{mK}$ bolometer plate was heated by micro-vibrations. The average amount of heat dissipated in the bolometer plate was around $10 \mathrm{nW}$ in the instrument tests and $40 \mathrm{nW}$ during some periods of the system tests at CSL. The heat inputs on the bolometer plate are discussed in the dilution cooler section below.

The basic characteristics of the $4 \mathrm{~K}$ cooler are summarized in Table 6. The sorption cooler cold head LVHX1 is on the $18 \mathrm{~K}$ plate of the HFI FPU, where the helium of the $4 \mathrm{~K}$ cooler is pre-cooled. The LFI reference loads (Bersanelli et al. 2010) on the $4 \mathrm{~K}$ box of the FPU are shown in Fig. 18. The positions of the thermometers and heaters on the FPU $4 \mathrm{~K}$ box are shown in Fig. 19. The heating belt of the $4 \mathrm{~K}$ PID is between the $70 \mathrm{GHz}$ reference loads and those for the $30 \mathrm{GHz}$ and $44 \mathrm{GHz}$ channels. The temperature stability is not as good for the latter as that obtained for the HFI horns and $70 \mathrm{GHz}$ loads. The two 
Table 6. Basic characteristics of the $4 \mathrm{~K}$ cooler.

\begin{tabular}{ll}
\hline \hline Working fluid & ${ }^{4} \mathrm{He}$ \\
Maximum cooling power at 17.5 K pre-cooling temperature & $19.2 \mathrm{~mW}$ \\
Required cooling power at 17.5 K pre-cooling temperature & $13.3 \mathrm{~mW}$ \\
Pre-cooling stages & Third V Groove 54 K Sorption cooler LR3 17.5-19 K \\
Nominal operating temperature & $4.5 \mathrm{~K}$ \\
Mass & \\
Compressors, pipes, cold stage, ... & $27.7 \mathrm{~kg}$ \\
Electronics and current regulator & $8.6 \mathrm{~kg}$ \\
Power & $120 \mathrm{~W}$ Max into current regulator \\
\hline
\end{tabular}

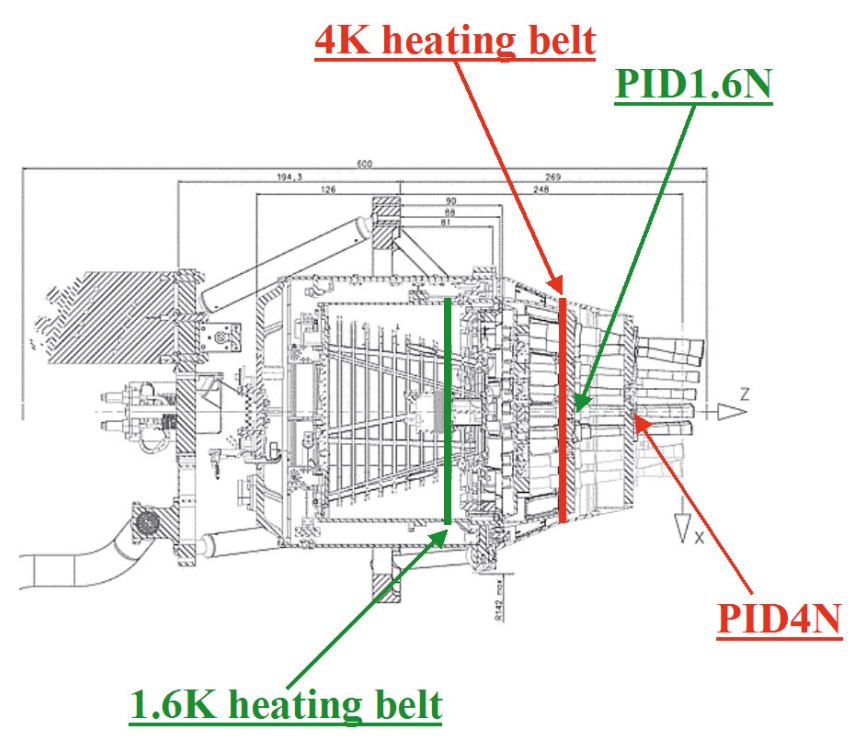

Fig. 19. View of the focal plane unit showing the thermal interfaces and the location of the heating belts and of the thermometers.

thermometers L1 and L2 monitor with high accuracy the temperature at this stage, which can be used to correct the $30 \mathrm{GHz}$ and $44 \mathrm{GHz}$ reference load signals.

\subsection{The dilution cooler}

The dilution cooler operates on an open circuit using a large quantity of ${ }^{4} \mathrm{He}$ and ${ }^{3} \mathrm{He}$ stored on board in four high pressure tanks. It includes a JT expansion valve producing cooling power for the $1.6 \mathrm{~K}$ stage of the FPU and pre-cooling for the dilution cooler. The microgravity dilution cooler principle was invented and tested by Benoît (Benoît et al. 1997) and developped into a space-qualified system by DTA Air Liquide (Triqueneaux et al. 2006). The gas from the tanks (at 300 bars at the start of the mission) is brought down to 19 bars through two pressure regulators, and the flows through the dilution circuit are regulated by a set of discrete restrictions, which can be chosen by telecommand.

The helium isotope flow rates for the different configurations of the restrictions are given in Table 7.

The heat lift margin HLm available for temperature regulation is determined by

- the He isotopes flow (HeFlow in $\mu \mathrm{mol} \mathrm{s}^{-1}$ ) given by the restriction configuration for $273 \mathrm{~K}$ temperature of the dilution cooler control unit (DCCU);

- the temperature of the DCCU $T_{\mathrm{DCCU}}$ in flight;
Table 7. Helium flow.

\begin{tabular}{lccc}
\hline \hline Flows & $\begin{array}{c}\text { flow }{ }^{4} \mathrm{He} \\
\mu \mathrm{mol} / \mathrm{s}\end{array}$ & $\begin{array}{c}\text { Ftot (DN3+DN4) } \\
\mu \mathrm{mol} / \mathrm{s}\end{array}$ & $\begin{array}{c}\text { flow }{ }^{3} \mathrm{He} \\
\mu \mathrm{mol} / \mathrm{s}\end{array}$ \\
\hline FMIN2 & 14.5 & 19.8 & 5.4 \\
FMIN & 16.6 & 22.9 & 6.3 \\
FNOM1 & 20.3 & 27.8 & 7.5 \\
FNOM2 & 22.6 & 30.8 & 8.2 \\
\hline
\end{tabular}

- the heat load from the bolometer plate at a temperature of $T_{\text {bolo }}$ and from the $1.6 \mathrm{~K} \mathrm{JT}$ stage at a temperature of $T_{\mathrm{JT} 1.6 \mathrm{~K}}$;

- the temperature of the dilution $T_{\text {dilu }}$.

This margin is given by

$$
\begin{aligned}
H L m[\mathrm{nW}]= & 3.2 \times 10^{-3} \text { HeFlow } \times T_{\text {dilu }}^{2} \times\left(T_{\mathrm{DCCU}} / 273-1\right)^{1.5} \\
& -250\left(T_{\mathrm{JT} 1.6 \mathrm{~K}}-1.28\right) \\
& -20\left(T_{\text {bolo }}-T_{\text {dilu }}\right) \\
& -490 \mathrm{nW}(\text { parasitic heat load }) .
\end{aligned}
$$

This allows for operation in flight at the lowest flow (FMIN2) at a temperature of $98 \mathrm{mK}$ and $108 \mathrm{nW}$ of power available for regulation, even at the highest temperature of the dilution panel in the spacecraft $\left(18^{\circ} \mathrm{C}\right)$. This should provide 32 months of operations after cool down.

\subsection{Thermal architecture}

In order to reach the required thermal stability on each thermal stage, a thermal architecture was designed on the $4 \mathrm{~K}, 1.6 \mathrm{~K}$ and $100 \mathrm{mK}$ stages (Piat et al. 2003). Temperature fluctuations are measured with very sensitive thermometers made of optimised NTD Ge (Piat et al. 2001, 2002) and readout by the same electronics as for the bolometers. Heating power could be applied with dedicated heaters controlled with a 24 bits DAC. Details on the temperature stability tests and results are given in Pajot et al. (2010).

\subsection{1. $4 \mathrm{~K}$ and 1.6 $\mathrm{K}$ stages}

The temperature of the $4 \mathrm{~K}$ box is regulated by a proportionalintegral-derivative (PID) servo system with a heating belt on the $4 \mathrm{~K}$ box providing a temperature stability so that the power spectrum temperature fluctuation is lower than $10 \mu \mathrm{K} \mathrm{Hz}^{-0.5}$ in the useful band of observation in Planck $(0.016 \mathrm{~Hz}$ to $100 \mathrm{~Hz})$ (Leroy et al. 2008).

An equivalent PID servo system controls the stability of the $1.6 \mathrm{~K}$ screen of the FPU, with a stability better than the 


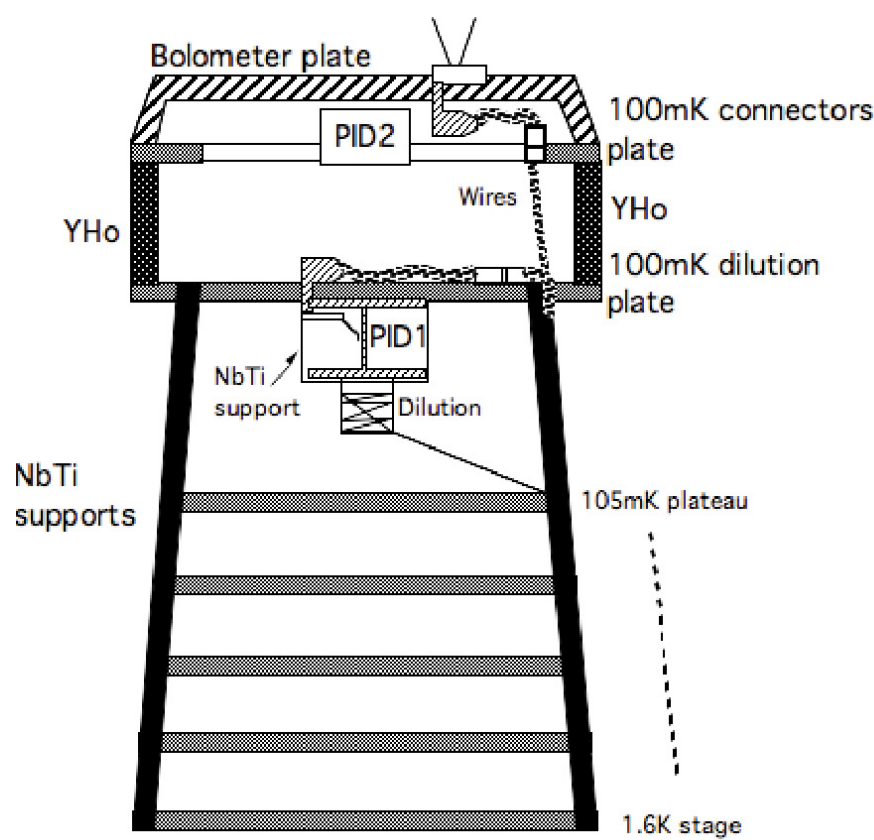

Fig. 20. Thermal architecture of the $100 \mathrm{mK}$ stage.

requirement of $28 \mu \mathrm{K} \mathrm{Hz}^{-0.5}$ (in the $0.016 \mathrm{~Hz}$ to $100 \mathrm{~Hz}$ frequency range). For both the $4 \mathrm{~K}$ and $1.6 \mathrm{~K}$ stages, the required stabilities were achieved during system tests as discussed in detail by Pajot et al. (2010).

\subsection{2. $100 \mathrm{mK}$ stage}

The temperature stability of the bolometer plate is achieved with both an active control and a passive thermal filter as shown in Fig. 20 (Piat 2000; Piat et al. 2000).

Two stages of PID regulation are included. The first one (PID1) is on the dilution itself and provides stability on long time scales. When no thermal perturbation is applied to the bolometer plate, PID1 alone provides the required stability. The second regulation system (PID2) is on the bolometer plate. The passive thermal filter is mounted between the dilution cold tip and the bolometer optical plate. The mechanical link between these two stages is built from a material made of an holmium-yttrium (HoY) alloy with high heat capacity at $100 \mathrm{mK}$. It gives a thermal time constant of several hours between these stages (Madet 2002).

The behaviour of the $100 \mathrm{mK}$ part of HFI was extensively modelled. The heat input expected on the bolometer plate are the input from the bias current of the bolometers (less than $1 \mathrm{nW}$ ), the microwave radiation reaching the bolometers $(0.12 \mathrm{nW})$, cosmic rays that penetrate the FPU box and deposit energy in the bolometer plate $(0.2 \mathrm{nW})$, the heat dissipated by microvibrations in the bolometer plate, and heating from the PID2 (about $1 \mathrm{mK}$ temperature increase for $30 \mathrm{nW}$ ).

Micro-vibrations from the $4 \mathrm{~K}$ compressors can add extra heating power on the $100 \mathrm{mK}$ bolometer plate (as was seen during the instrument and system tests). The average amount of heat dissipated in the bolometer plate was around $10 \mathrm{nW}$ in the instrument tests and $40 \mathrm{nW}$ during some periods of the system tests at CSL.

Figure 21 shows an example of the predicted gradients on the bolometer plate when the PID is off. The three HoY links to the dilution cooler are seen as the blue (cold) spots through which the heat from the cosmic rays and bolometer bias current

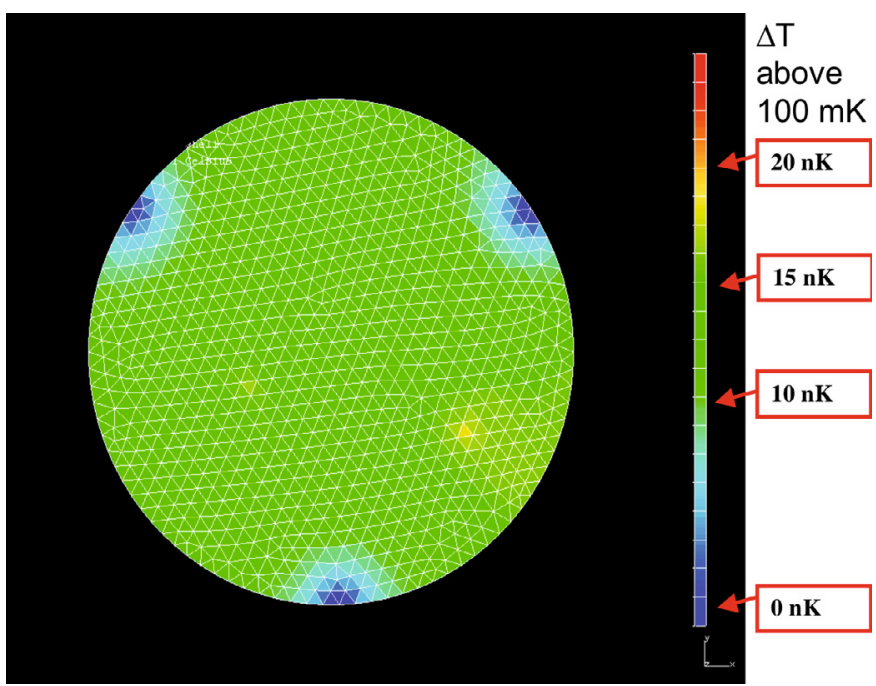

Fig. 21. Simulation of the gradients induced on the bolometer plate by particles when the PID is off.

dissipation is transferred towards the dilution stage. The yellow areas are due to recent cosmic rays hits. If the PID2 is turned on with a typical heat input of $100 \mathrm{nW}$, the gradients will be 300 times steeper. When the heat input varies, the top of the HoY feet will readjust very slowly because of their long time constants. The gradient will change accordingly. It is thus believed that using the PID2 will not improve the temperature stability of the bolometer plate for rapid changes of heat inputs. The slow drifts are damped by PID1 on the dilution plate. Thus PID2 is considered as a back-up of PID1 and will not be used in flight.

The main temporary perturbations on the $100 \mathrm{mK}$ stage should come from solar flares. Only a few events at most are expected during the mission leading to the loss of a few days of operation of the dilution cooler.

\section{Model philosophy and calibrations}

\subsection{Model philosophy}

The Planck spacecraft (S/C) development philosophy was based on two models, the qualification model and the Planck Flight model (equipped with the flight models of the instruments). The HFI followed the same approach. The testing programme of the $\mathrm{S} / \mathrm{C}$ imposed a cryogenic verification model in addition to the usual space qualification. This was called the Cryogenic Qualification Model (CQM). The S/C CQM was required to be identical to the PFM for the cold part (PPLM) and similar for the SVM. The goal was to verify as far as necessary the concept of the entire cryogenic chain and associated ancillary units. The HFI model philosophy, numbering and naming were the same, with a CQM HFI partly composed of flight units, or candidates to be swapped with flight ones later on or to be used as spare units for the PFM. The qualification of HFI followed a standard testing sequence for the warm units in compliance with the environmental conditions imposed by the mission through the $\mathrm{S} / \mathrm{C}$ interfaces. A major step for the HFI was the performance verification of the FPU including the cold parts of the $1.6 \mathrm{~K}$ and $100 \mathrm{mK}$ of the dilution cooler, equipped with a limited number of optical channels. This test allowed the validation and the characterization of the whole detection chain in a thermal configuration identical to the flight one, i.e. bolometers at $100 \mathrm{mK}$, cold filters at $100 \mathrm{mK}, 1.6 \mathrm{~K}$, and $4 \mathrm{~K}$, JFET preamplifier at $110 \mathrm{~K}$, and 
with warm preamplifier and read-out Electronics delivering the digital signal through the DPU to the electrical ground support equipment (EGSE). It also provided an opportunity to test the dilution cooler in conditions similar to those of the PFM. In addition, it was the first complete test for the calibration facility and offered excellent preparation for the calibration of the PFM.

Once the HFI was integrated in the spacecraft, the most important test of the qualification, acceptance and verification process was the cryogenic test performed in the Focal $\mathrm{V}$ vacuum chamber of the Centre Spatial de Liège in Belgium. The CQM demonstrated the capability of HFI to reach the objectives of the mission, i.e. meeting the major required characteristics, like the photometric sensitivity and the lifetime of the dilution cooler. The test of the complete cryogenic chain was successfully completed at the PFM. The dilution cooler, $4 \mathrm{~K}$ cooler, $18 \mathrm{~K}$ sorption cooler and the passive radiators were all mounted in the flight configuration. A large number of procedures for the flight were successfully tested in preparation to the cool down, commissioning and flight operations.

\subsection{HFI calibration philosophy}

The cryo-qualification model (CQM) and the proto-flight model (PFM) of the HFI were extensively tested and calibrated on the ground (Pajot et al. 2010). In parallel, numerical models of the instrument and of its response (Catalano 2008) were developped to interpret the test data and to check that the obtained results were consistent with those expected, thereby validating the operation of the instrument. At the same time, deviations from the models alerted us to unanticipated behaviour, like the low frequency excess response of the bolometers. Finally, the instrument models can be used to interpolate ground calibration results into the phase space of operating parameters that were not explored finely enough during tests. The final calibration will rely on data obtained in-flight, as far as possible. Some ground calibration data, like the spectral transmissions, will remain the reference (Pajot et al. 2010).

\subsection{Schedule}

The development and testing of HFI was complex, and so the two models were delivered in groups of functional units on time to be integrated on the $\mathrm{S} / \mathrm{C}$. The main dates in the history of HFI were

- CQM delivery of the units in 2004;

- integration on the CQM S/C from end of 2004 to mid 2005;

- cryotest at CSL June to September 2005;

- PFM delivery of units from mid 2006 to mid 2007;

- integration of both LFI and HFI FPUs at the end of 2006;

- integration with the $\mathrm{S} / \mathrm{C}$ and environment tests up to March 2008;

- cryogenic test at CSL from June to September 2008.

The S/C was then finally tested and prepared for the transportation to Kourou for the launch. The last ground operations on HFI were mixed with regular health verifications, the cleaning of the $4 \mathrm{~K}$ cooler during $120 \mathrm{~h}$, the purge of the pipes of the dilution cooler and the filling of the ${ }^{3} \mathrm{He}$ and ${ }^{4} \mathrm{He}$ tanks.

\subsection{Management}

Following the selection of the Planck mission, the LFI and HFI instruments, each led by a principal investigator (PI), were selected for flight. Each PI had full responsibility for the delivery of their respective instrument. HFI was built by a consortium of institutes from France, Italy, Spain, the UK and the USA. A work-breakdown structure defined the work to be done up to the completion of the instrument. In each institute a coinvestigator (Co-I) and an element manager were responsible for the delivery of their specific equipment. The industry often participated as a subcontractor. Funding was provided by national agencies, and the relationship between the PI and the Co-Is is defined in a Memorandum of Understanding, which is the reference document in the work and responsibilities on the project.

The HFI project is organized around the PI with key persons like the Co-PI, who supervises the data processing, the instrument scientist responsible for the instrument, and the scientist in charge of calibrations and operations. The technical team is organized around the project manager with a project controller, a system engineer, a manager responsible for integration verification and test (AIV-AIT), a product assurance manager, and a team of architects responsible for the various engineering tasks like cryogenics and thermics, mechanics, optics, EMI-EMC. The technical team also includes engineers and scientists responsible for the delivery of identified sub-systems: the dilution cooler, the $4 \mathrm{~K}$ cooler, readout electronics, and the DPU. The integration and system tests were managed by the AIV-AIT manager with a team of specialists. They also operate during the AIT phase on the $\mathrm{S} / \mathrm{C}$ and in Kourou.

\section{Conclusions}

The ambition of the HFI instrument is to provide a new picture of the CMB with much improved sensitivity, high angular resolution and a low level of systematic effects. It will map the sky in the sub-THz range with sensitivity limits near to the fundamental fluctuations of the observed radiation itself. The calibration and tests on the ground indicate that this goal should indeed be achieved in flight, with a sensitivity to within a factor of two of the photon noise in all channels (i.e. in line with the goal sensitivity announced in the instrument proposal) and about two times better than required by the scientific goals. This performance was achieved by experts in all of the relevant instrumental fields working together and agreeing on goals that were close to the limit of what could be achieved in the time-frame of this project. The preliminary concepts defining the architecture and the specification of sub-systems provided a robust design that could be adapted to cope with some variability of individual components. Some progress in component design can be expected for future CMB experiments. However, the experience of the HFI suggests that significantly higher sensitivities can only be achieved by using much larger numbers of detectors.

Careful attention was paid to reduce systematic effects to levels commensurate with the high sensitivity of the HFI. This required substantial efforts in the optical and electrical designs. The instrument was carefully calibrated during tests on the ground, though there remain a few uncertainties as described in this paper. Some systematic effects could be identified and measured during tests of the HFI, providing the HFI data processing centre with a wealth of information for future data reduction. Together with the Low Frequency Instrument, the HFI should be able to produce a new picture of the CMB and of the submillimetre sky.

Acknowledgements. The Planck-HFI instrument (http://hfi.planck.fr/) was designed and built by an international consortium of laboratories, universities and institutes, with important contributions from the industry, under the 
leadership of the PI institute, IAS at Orsay, France. It was funded in particular by CNES, CNRS, NASA, STFC and ASI. The authors extend their gratitude to the numerous engineers and scientists, who have contributed to the design, development, construction or evaluation of the HFI instrument. The authors are pleased to thank the referee for his/her very useful remarks.

\section{References}

Ade, P. A. R., Savini, G., Sudiwala, R., et al. 2010, A\&A, 520, A11 Baranov, V. K., \& Mel'nikov, G. K. 1966, Sov. J. Opt. Technol., 33, 408

Benoît, A., Sirbi, A., Bradshaw, T., et al. 1997, in Sixth European Symposium on Space Environmental Control Systems, ed. T.-D. Guyenne, ESA SP, 400, 497

Benoît, A., Zagury, F., Coron, N., et al. 2000, A\&AS, 141, 523

Benoît, A., Ade, P. A. R., Amblard, A., et al. 2002, Astroph. Phys., 17, 101

Bersanelli, M., Mandolesi, N., Butler, R. C., et al. 2010, A\&A, 520, A4

Bock, J. J., Chen, D., Mauskopf, P. D., \& Lange, A. E. 1995, Space Sci. Rev., 74,229

Bouchet, F. R., Gispert, R., \& Puget, J.-L. 1996, in AIP Conf. Ser. 348, ed. E. Dwek, 255

Bradshaw, T. W., \& Orlowska, A. H. 1997, in Sixth European Symposium on Space Environmental Control Systems, ed. T.-D. Guyenne, ESA SP, 400, 465 Brienza, D., de Angelis, L., de Bernardis, P., et al. 2006, in Seventh International Workshop on Low Temperature Electronics, WOLTE-7, ESA-WPP-264, 283

Catalano, A. 2008, Ph.D. Thesis, École doctorale Astronomie et Astrophysique d'Ile-de-France

Chanin, G., \& Torre, J.-P. 1984, J. Opt. Soc. Am. A, 1, 412

Crill, B. P., Ade, P. A. R., Artusa, D. R. et al. 2003, ApJS, 148, 527

Doucerain, C., Lamarre, J.-M., \& Torre, J.-P. 1995, in Workshop on bolometers for millimetre and submillimetre space projects, Orsay, 15-16 June

Dupac, X., \& Tauber, J. 2005, A\&A, 430, 363

Gaertner, S., Benoît, A., Lamarre, J.-M., et al. 1997, A\&AS, 126, 151

Haller, E. E., Itoh, K. M., \& Beeman, J. W. 1996, in Submillimetre and FarInfrared Space Instrumentation, ed. E. J. Rolfe, \& G. Pilbratt, ESA SP, 388, 115

Hanany, S., Jaffe, A. H., \& Scannapieco, E. 1998, MNRAS, 299, 653

Harper, D. A., Hildebrand, R. H., Stiening, R., \& Winston, R. 1976, Appl. Opt., 15,53

Holmes, W. A., Bock, J. J., Crill, B. P. et al. 2008, Appl. Opt., 47, 5996

Holzapfel, W. L., Wilbanks, T. M., Ade, P. A. R., et al. 1997, ApJ, 479, 17

Jones, R. C. 1953, J. Opt. Soc. Am. (1917-1983), 43, 1

Jones, W. C., Bhatia, R., Bock, J. J., \& Lange, A. E. 2003, in SPIE Conf. Ser. 4855, ed. T. G. Phillips, \& J. Zmuidzinas, 227

Kraus, J. D. 1966, Radio Astronomy (New York: McGraw-Hill Book Co.)

Lamarre, J.-M. 1986, Appl. Opt., 25, 870

Lamarre, J.-M., Désert, F.-X., \& Kirchner, T. 1995, Space Sci. Rev., 74, 27

Lamarre, J.-M., Puget, J.-L., Bouchet, F., et al. 2003, New Astron. Rev., 47, 1017

Lee, A. T., Ade, P. A. R., Balbi, A., et al. 1999, in AIP Conf. Ser., 476, 224, 3 K cosmology, ed. L. Maiani, F. Melchiorri, \& N. Vittorio

Leroy, C., Maisonneuve, M., Piat, M., et al. 2008, in SPIE Conf. Ser., 7017, 701713

Madet, K. 2002, Ph.D. Thesis, Université Joseph Fourier (Grenoble 1)

Maffei, B., Noviello, F., Murphy, J., et al. 2010, A\&A, 520, A12

Mandolesi, N., Smoot, G. F., \& Bersanelli, M. 1994, in Present and Future of the Cosmic Microwave Background, ed. J. L. Sanz, E. Martinez-Gonzalez, \& L. Cayon (Berlin: Springer Verlag), Lecture Notes Physics, 429, 228

Mandolesi, N., Bersanelli, M., Butler, R., et al. 2010, A\&A, 520, A3

Masi, S., Ade, P. A. R., Bock, J. J., et al. 2006, A\&A, 458, 687

Mather, J. C. 1982, Appl. Opt., 21, 1125

Mather, J. C., Cheng, E. S., Eplee, Jr., R. E., et al. 1990, ApJ, 354, L37

Mauskopf, P. D., Bock, J. J., del Castillo, H., Holzapfel, W. L., \& Lange, A. E. 1997, Appl. Opt., 36, 765

Murphy, J. A., Colgan, R., Gleeson, E., et al. 2002, in Experimental Cosmology at Millimetre Wavelengths, ed. M. de Petris, \& M. Gervasi, AIP Conf. Ser., 616, 282

Murphy, J., Peacocke, T., Maffei, B., et al. 2010, J. Inst., accepted

Pajot, F., Ade, P. A. R., Beney, J.-L., et al. 2010, A\&A, 520, A10

Piacentini, F., Ade, P. A. R., Bhatia, R. S., et al. 2002, ApJS, 138, 315

Piat, M. 2000, Ph.D. Thesis, Université de Paris-Sud (Paris XI)

Piat, M., Leriche, B., Torre, J.-P., et al. 2000, Nucl. Instrum. Methods Phys. Res. A, 444,413
Piat, M., Torre, J.-P., Lamarre, J.-M., Beeman, J. W., \& Bhatia, R. S. 2001, J. Low Temperature Phys., 125

Piat, M., Torre, J.-P., Lamarre, J.-M., et al. 2002, Low Temperature Detectors, 605,79

Piat, M., Lamarre, J.-M., Meissonnier, J., et al. 2003, in SPIE Conf. Ser. 4850, ed. J. C. Mather, 740

Rieke, F. M., Lange, A. E., Beeman, J. W., \& Haller, E. E. 1989, IEEE Trans. Nucl. Sci., 36, 946

Rosset, C., Tristram, M., Ponthieu, N., et al. 2010, A\&A, 520, A13

Smoot, G. F., Bennett, C. L., Kogut, A., et al. 1991, ApJ, 371, L1

Tauber, J. A., Mandolesi, N., Puget, J.-L., et al. 2010a, A\&A, 520, A1

Tauber, J. A., Norgaard-Nielsen, H., Ade, P. A. R., et al. 2010b, A\&A, 520, A2

The Planck consortium 2005, PLANCK - The Scientific Programme (The Planck Bluebook), ESA-SCI(2005)1

Triqueneaux, S., Sentis, L., Camus, P., Benoît, A., \& Guyot, G. 2006, Cryogenics, 46, 288

Tristram, M. 2005, Ph.D. Thesis, Université Joseph Fourier, Grenoble 1

Vaillancourt, J. E. 2005, Rev. Sci. Instrum., 76, 043107

Welford, W. T., \& Winston, R. 1978, The optics of nonimaging concentrators, Light and solar energy (Academic Press)

Woodcraft, A. L., Sudiwala, R. V., Ade, P. A. R., et al. 2003, Appl. Opt., 42, 5009

Yvon, D., Panh, J., Landé, J., et al. 2008, J. Low Temperature Phys., 151, 448

1 Laboratoire d'Étude du Rayonnement et de la Matière en Astrophysique (LERMA), Observatoire de Paris, ENS, UPMC, UCP, CNRS, 61 avenue de l'Observatoire, 75014 Paris, France e-mail: jean-michel.lamarre@obspm.fr

2 IAS, Institut d'Astrophysique Spatiale, CNRS \& Université Paris 11, Bâtiment 121, 91405 Orsay, France

3 Cardiff University, School of Physics and Astronomy, The Parade, Cardiff CF24 3AA, UK

4 Institut d'Astrophysique de Paris, UMR 7095, CNRS and Université Pierre \& Marie Curie-Paris 6, 98 bis boulevard Arago, 75014 Paris, France

5 Department of Physics, California Institute of Technology, Mail code: 59-33, Pasadena, CA 91125, USA

6 Jet Propulsion Laboratory, California Institute of Technology, 4800 Oak Grove Drive, Pasadena, CA 91109, USA

7 European Space Agency - ESTEC, Astrophysics Division, Keplerlaan 1, 2201 AZ Noordwijk, The Netherlands

${ }^{8}$ LAL, Laboratoire de l'Accélerateur Linéaire, CNRS \& Université Paris 11, Bâtiment 200, 91898 Orsay Cedex, France

9 Institut Néel, CNRS, Univ. Joseph Fourier Grenoble I, 25 rue des Martyrs, BP 166, 38042 Grenoble Cedex 9, France

10 CESR, Centre d'Étude Spatiale des Rayonnements, CNRS, 9 Av. du colonel Roche, BP44346, 31038 Toulouse Cedex 4, France

11 CNES, 18 avenue Edouard Belin, 31401 Toulouse Cedex 9, France

12 Laboratoire Astroparticule et Cosmologie (APC), CNRS \& Université Paris Diderot - Paris 7, 10 rue A. Domon et L. Duquet, 75205 Paris Cedex 13, France

13 STFC, Rutherford Appleton Laboratory, Harwell Science and Innovation Campus, Didcot, OX11 0QX, UK

14 Kavli Institute for Particle Astrophysics and Cosmology and Department of Physics, Stanford University, 382 via Pueblo Mall, Stanford, CA 94305, USA

15 Dipartimento di Fisica, Universitá La Sapienza, Piazzale Aldo Moro 2, 00185 Roma, Italy

16 Laboratoire d'Astrophysique Observatoire de Grenoble (LAOG), CNRS, BP 53, 38041 Grenoble Cedex 9, France

17 European Space Agency - ESAC, PO box 78, 28691 Villanueva de la Cañada, Madrid, Spain

18 Institute of Astronomy, University of Cambridge, Madingley Road, Cambridge CB3 OHA, UK

19 Princeton University, Department of Physics, Joseph Henry Laboratory, USA 
20 Laboratoire de Physique Subatomique et de Cosmologie (LPSC), Univ. Joseph Fourier Grenoble I, CNRS/IN2P3, Institut National Polytechnique de Grenoble, 53 Avenue des Martyrs, 38026 Grenoble Cedex, France

21 The University of Manchester, JBCA, School of Physics and Astronomy, Manchester M13 9PL, UK

22 Department of Experimental Physics, National University of Ireland (NUI), Maynooth, Co. Kildare, Ireland
23 Optical Science Laboratory, University College London (UCL), Gower Street, WC1E 6BT London, UK

${ }^{24}$ SUPA, Institute of Astronomy, University of Edinburgh, Blackford Hill, Edinburgh EH9 3HJ, UK

25 Institute of Radiophysics and Electronics, NAS of Ukraine, 12 Proskura St., 61085 Kharkov, Ukraine

26 CEA, CE Saclay, IRFU/Service de Physique des Particules, 91191 Gif-sur-Yvette Cedex, France 